\title{
Nonsinglet polarized nucleon structure function in infrared-safe QCD
}

\author{
Leila Ghasemzadeh, ${ }^{1, *}$ Abolfazl Mirjalili $\odot,{ }^{1, \dagger}$ and S. Atashbar Tehrani ${ }^{2, \ddagger}$ \\ ${ }^{1}$ Physics Department, Yazd University, P.O. Box 89195-741, Yazd, Iran \\ ${ }^{2}$ School of Particles and Accelerators, Institute for Research in Fundamental Sciences (IPM), \\ P.O. Box 19395-5531, Tehran, Iran
}

(Received 18 April 2019; revised manuscript received 28 October 2019; published 10 December 2019)

The polarized nucleon structure function in the nonsinglet case is investigated here by a new insight rather than conventional perturbative QCD (pQCD). For this purpose, we note that the solution of the evolution equations in moment space involves noninteger powers of the coupling constant. Therefore, it is possible to employ a new approach which is called the fractional analytical perturbation theory. Consequently, it is possible to remove the Landau singularities of the renormalized coupling, i.e., at the scales $Q \sim \Lambda$, using this approach. This provides an opportunity to continue the desired calculations toward small values of energy scales even less than the $\Lambda$ scale. To modify the analytical perturbation theory, a newer approach is introduced, called $2 \delta \mathrm{anQCD}$, in which the spectral function of the holomorphic coupling is parameterized in the low-energy region by two delta functions. This model gives us more reliable results for the considered QCD observables, even in the deep infrared region. We calculate the nonsinglet part of the polarized nucleon structure function, using the $2 \delta$ anQCD model, and compare it with the result from the underlying pQCD where both are in a new defined scheme, called the Lambert scheme. For this purpose, we employ the anQCD package in the Mathematica environment to establish the analytic (holomorphic) coupling constant. The results at various energy scales are also compared with the available experimental data, and it turns out that there is a good consistency between them. The results show that the obtained nucleon structure function at small energy scales has smoother behavior when using the $2 \delta \mathrm{anQCD}$ model than the underlying pQCD. In fact, the coupling constant in analytic QCD behaves moderately, and it makes the result approach the available data in a better way. We also consider the $Q^{2}$ dependence of the Bjorken sum rule (BSR), using the $2 \delta$ anQCD model. There is a good agreement between the available experimental data for the BSR and the results from the utilized model, especially at low-energy scales.

DOI: $10.1103 /$ PhysRevD.100.114017

\section{INTRODUCTION}

QCD analysis of deep-inelastic scattering (DIS) data provides us new insight into the hadron physics. The reliability of theoretical results can be increased by testing the analysis, for instance, of a hard lepton scattering off the hadrons. A reliable description of DIS at large momentum transfer $Q^{2} \gg 1 \mathrm{GeV}^{2}$ can be obtained, considering the twist expansion and factorization theorem. Following that, one can use the DIS processes to better understand the nucleon structure function. In this regard, the polarized and unpolarized structure functions can be first modeled, using

\footnotetext{
*Leilaghasemzadeh@stu.yazd.ac.ir

Corresponding author.

A.Mirjalili@yazd.ac.ir

*Atashbar@ipm.ir

Published by the American Physical Society under the terms of the Creative Commons Attribution 4.0 International license. Further distribution of this work must maintain attribution to the author(s) and the published article's title, journal citation, and DOI. Funded by SCOAP .
}

different parametrization methods. Then they can be evolved to high-energy scales, employing the QCD evolution equations. During the evolution process, the numerical values of structure functions are affected by the energy scale, at which the renormalized coupling constant as the perturbation expansion parameter is evaluated. On the other hand, the analysis of the structure functions should lead to consistent results with the related experimental data. To achieve this aim, it is needed to model the parton densities, as ingredients of nucleon structure, at an initial energy scale. A further step is the evolution of these densities to high-energy scales. Therefore, the role of the renormalized coupling constant $\alpha_{s}\left(Q^{2}\right)$ is important and should be considered in detail.

At low- $Q^{2}$ energy scales, below 1 or $2 \mathrm{GeV}^{2}$, the qualification of DIS as a hard process is not appropriate. Its analysis is faced with two essential difficulties in the low- $Q^{2}$ region. One of them is related to the higher-twist corrections which are dominantly affecting the contribution of the leading twist at low values of the energy scale. Therefore, the twist corrections at higher orders in $\alpha_{s}$ but at 
a low-energy scale causes the first difficulty. In addition, since $\alpha_{s}\left(Q^{2}\right)$ as the QCD running coupling grows rapidly at the energy scales near the Landau singularities, the result of pQCD would not be reliable and this represents the second difficulty.

A new approach that is called the analytic perturbation theory (APT) can be applied to resolve these problems. This approach has been developed by Shirkov, Solovtsov, et al. [1-6]. There, the running QCD coupling of conventional pQCD is transformed into an analytic (holomorphic) function of $Q^{2}$ which is accordingly called the APT coupling constant. In this regard, one needs a dispersion relation, involving a spectral or discontinuity function which is the imaginary part of the coupling along the timelike semiaxis $\left(Q^{2}<0\right)$ in the complex $Q^{2}$ plane. In the framework of APT, the images (analogs) which correspond to integer powers of the original perturbative QCD (pQCD) coupling constant can also be constructed. Later on, an extension to the analogs of noninteger powers was performed, which is called fractional APT (FAPT). Some newer constructions of the coupling constant in analytic QCD (anQCD) are based on the modifications of the spectral function at low-energy scales. In the case of this modification, the spectral function is constructed in a specific manner, by parameterizing its behavior in the low-energy region by two positive delta functions $[7,8]$. The presented results of this paper are based on employing this model which is called $2 \delta$ anQCD.

We remark that anQCD is not the only approach to obtain a proper behavior of the coupling constant at a low energy, i.e., at infrared (IR) scales. To achieve an IR-safe QCD description, one can also refer specifically to the AdS/CFT coupling of Brodsky, de Teramond, and Deur [9] or the dispersive approach of Dokshitzer et al. [10,11]. A new review of the QCD running coupling in various approaches can be found in Ref. [12].

The remainder of this paper consists of the following sections. In Sec. II, we provide a brief discussion of the theoretical formalism for different aspects of anQCD, including the $2 \delta$ anQCD model. In Sec. III, we deal with the theoretical formalism of pQCD in DIS processes, where the Jacobi polynomials and the rational expansion for the moment of polarized nucleon structure function are also presented. In Sec. IV, we employ the $2 \delta$ anQCD model as the approach to evaluate the perturbation expansions and to extract the moments of the polarized structure functions at the next-to-leading-order (NLO) approximation. In Sec. V, we also utilize this model to evaluate the Bjorken sum rule for polarized nucleon structure functions. Finally, we give our summary and conclusions in Sec. VI.

\section{AN OVERVIEW OF ANALYTIC QCD APPROACHES}

The anQCD approaches are intended to address the problems which were mentioned in the introduction, especially the second problem, i.e., the presence of the Landau singularities in pQCD couplings $\alpha_{s}\left(Q^{2}\right)$. FAPT [1-6,13-17] is one such approach. Other approaches [9-11] could be applied, but we do not follow this line here. The calculations in the present work will be performed using the $2 \delta \mathrm{anQCD}$ approach $[7,8]$, which has significant modifications with respect to FAPT.

For this purpose, we first give a brief description of (F)APT, a description essentially using Ref. [18]. After that, we give a summarized description of the $2 \delta$ anQCD model.

In the FAPT approach, the running pQCD coupling $a_{s}\left(Q^{2}\right) \equiv \alpha_{s}\left(Q^{2}\right) / 4 \pi$ of $\mathrm{pQCD}$ is transformed into a holomorphic function of $Q^{2}$ in which first the conversion $a\left(Q^{2}\right) \mapsto \mathcal{A}_{1}\left(Q^{2}\right)$ is performed for the original pQCD coupling. The new coupling $\mathcal{A}_{1}\left(Q^{2}\right)$ is an analytic (holomorphic) function of $Q^{2}$ in the complex $Q^{2}$ plane with the only exception of the timelike semiaxis $\left(Q^{2} \leq 0\right)$, and it is called the APT coupling constant.

As it will be seen later on, the APT coupling is given by a dispersion relation. In this relation, there is the pQCD spectral density, denoted by $\rho^{(\mathrm{pt})}(\sigma) \equiv \operatorname{Im} \alpha_{s}\left(Q^{2}=\right.$ $-\sigma-i \epsilon) / \pi$, which in APT is not changed in the complex $Q^{2}$ plane for the whole negative axis, given by the condition $\sigma \geq 0$. For the unphysical cut, i.e., $0<Q^{2}<\Lambda^{2}$, this spectral function is set equal to zero [18]. Following the same dispersion relation, based on the APT framework, the images (analogs) $\mathcal{A}_{n}\left(Q^{2}\right)$ of integer powers $a^{n}\left(Q^{2}\right)$ can also be constructed.

In contrast with the original $a^{n}\left(Q^{2}\right)$, the couplings $\mathcal{A}_{n}\left(Q^{2}\right)$ are changing slowly at low $Q^{2}$ values. On the other hand, at large $Q^{2}$ values, these two couplings are close to each other $\left[\mathcal{A}_{1}\left(Q^{2}\right)-a\left(Q^{2}\right) \sim \Lambda_{\mathrm{QCD}}^{2} / Q^{2}\right]$.

The FAPT approach, which was summarized here, is an extension to noninteger powers $\nu$ in which $a^{\nu}$ maps to $\mathcal{A}_{\nu}$ [13-17]. This case has many applications to DIS processes. One can refer to Ref. [19], which contains reasonable results in analyzing with the FAPT approach the DIS data for the hadron characteristics. Alongside the FAPT framework, there are various analytic QCD models which can be found in Refs. [18,20-28]. One of them is called the $2 \delta$ anQCD model $[7,8]$, which will be discussed later on in this section.

In the framework of the FAPT approach, one can obtain the following dispersion relation for the analogs (images) $\mathcal{A}_{\nu}^{(l)}$ of the running coupling $a^{\nu}\left(Q^{2}\right) \equiv\left(\alpha_{s}\left(Q^{2}\right) / \pi\right)^{\nu}$ at the $l$-loop order in the spacelike domain, by applying the Cauchy theorem [1-6]:

$\mathcal{A}_{\nu}^{(l)}(L)=\int_{0}^{\infty} \frac{\rho_{\nu}^{(l)}[\sigma]}{\sigma+Q^{2}} d \sigma=\int_{-\infty}^{\infty} \frac{\rho_{\nu}^{(l)}\left(L_{\sigma}\right)}{1+\exp \left(L-L_{\sigma}\right)} d L_{\sigma}$.

In Eq. (1), $L_{\sigma}=\ln \left(\sigma / \Lambda^{2}\right)$ and $L=\ln \left(Q^{2} / \Lambda^{2}\right)$. The result of Eq. (1) does not contain any Landau singularities. 
The spectral function $\rho_{\nu}^{(l)}$ in Eq. (1) has the following representation [1-6]:

$$
\rho_{\nu}^{(l)}\left(L_{\sigma}\right) \equiv \frac{1}{\pi} \operatorname{Im}\left(a_{(l)}^{\nu}(L-i \pi)\right)=\frac{\sin \left[\nu \varphi_{(l)}(L)\right]}{\pi\left(R_{(l)}(L)\right)^{\nu}},
$$

where

$$
\begin{aligned}
& R_{(l)}(L)=\left|a_{(l)}(L-i \pi)\right|, \\
& \varphi_{(l)}(L)=\arg \left(a_{(l)}(L-i \pi)\right) .
\end{aligned}
$$

Considering Eq. (2) together with Eq. (1), it is seen that the images $\mathcal{A}_{\nu}$ do not obey the standard algebra such that $\mathcal{A}_{\nu} \mathcal{A}_{\mu} \neq \mathcal{A}_{\nu+\mu}$.

It can be proved that, at the one-loop approximation, $\varphi_{(1)}$ and $R_{(1)}$ in Eq. (2) are the simplest and are given, respectively, by [18]

$$
\begin{aligned}
& \varphi_{(1)}(L)=\arccos \left(\frac{L}{\sqrt{L^{2}+\pi^{2}}}\right), \\
& R_{(1)}(L)=\beta_{0} \sqrt{L^{2}+\pi^{2}} .
\end{aligned}
$$

One can reproduce at $Q^{2}=0$ the maximum value for $\mathcal{A}_{1}^{(1)}(L)$, i.e., $\mathcal{A}_{1}^{(1)}(L=-\infty)$, which can be shown to have the well-known expression [1]

$$
\mathcal{A}_{1}^{(1)}(-\infty)=\int_{-\infty}^{\infty} \frac{d L_{\sigma}}{\beta_{0}\left(L_{\sigma}^{2}+\pi^{2}\right)}=\frac{1}{\beta_{0}}>\mathcal{A}_{1}^{(1)}(L) .
$$

For this purpose, it is needed to substitute Eq. (4) in Eq. (2) and then insert in Eq. (1) the result for $\rho_{1}^{(1)}$.

The situation becomes more complicated when the twoloop approximation is considered. In this case, the solution of the QCD- $\beta$ function will yield the following result for $a_{s(2)}$ in terms of the Lambert- $W$ function [18]:

$$
a_{s(2)}=-\frac{1}{c_{1}} \frac{1}{1+W_{-1}\left(z_{W}(L)\right)} .
$$

In this equation, $z_{W}(L)$ is given by

$$
z_{W}(L)=-c_{1}^{-1} e^{-1-L \beta_{0} / c_{1}},
$$

where $\quad c_{1}=\beta_{1} / \beta_{0}=\left(102-38 N_{f} / 3\right) /\left(11-2 N_{f} / 3\right) / 4$, in which $N_{f}$ is denoting the number of active quark flavors and $Q^{2}=\left|Q^{2}\right| \exp (i \phi)$ is considered to have $0 \leq \phi<\pi$.

Considering Eq. (3), the required expressions for $R_{(2)}$ and $\varphi_{(2)}$ would be, respectively,

$$
\begin{aligned}
& R_{(2)}(L)=c_{1}\left(N_{f}\right)\left|1+W_{-1}\left(z_{W}(L+i \pi)\right)\right|, \\
& \varphi_{(2)}(L)=\arccos \left[\frac{\operatorname{Re}\left(1+W_{-1}\left(z_{W}(L+i \pi)\right)\right)}{R_{(2)}(L)}\right] .
\end{aligned}
$$

Here $W_{-1}(z)$ represents the appropriate branch of the Lambert- $W$ function. Extension of the calculations up to the four-loop level can be found in Ref. [29].

Because of the linearity of the transforms in Eq. (1), there is a one-to-one correspondence between the pQCD and FAPT expansion [5]. This linearity can be exposed, considering single-scale quantity $D\left(Q^{2}, \mu_{R}^{2}\right)$ at the renormalization scale $\mu_{R}^{2}=Q^{2}$ within the minimal subtraction scheme. In this regard, the expansions for $D$ and its corresponding image, i.e., $\mathcal{D}$, have the following expansions [18]:

$$
\text { pQCD: } D\left(Q^{2}\right)=d_{0} a^{\nu_{0}}\left(Q^{2}\right)+\sum_{n} d_{n} a^{n+\nu_{0}}\left(Q^{2}\right),
$$

FAPT: $\mathcal{D}\left(Q^{2}\right)=d_{0} \mathcal{A}_{\nu_{0}}\left(Q^{2}\right)+\sum_{n} d_{n} \mathcal{A}_{n+\nu_{0}}\left(Q^{2}\right)$.

We note that $d_{i}$ coefficients are identical in both expansions, and the renormalization scale $\mu_{R}^{2}=Q^{2}$ is used.

After the summarized description of the FAPT approach, we now present briefly the other anQCD model, which at low-energy scales (where Landau singularities appear in a) has a modified spectral function. It is therefore expected that, by applying this model, more reliable results are obtained, arising out of the anQCD approach. This model, as we mentioned earlier, is called $2 \delta$ anQCD. Our calculations in this paper are entirely based on utilizing this model.

In $2 \delta$ anQCD $[7,8]$, the spectral function $\rho(\sigma) \equiv$ $\operatorname{Im} \mathcal{A}_{1}(-\sigma-i \epsilon)$ is equal to the spectral function $\rho^{(\mathrm{pt})}(\sigma) \equiv$ $\operatorname{Im} a(-\sigma-i \epsilon)$ of the underlying pQCD coupling $a$ only for $\sigma \geq M_{0}^{2}$, where $M_{0}^{2} \sim 1 \mathrm{GeV}^{2}$ is regarded as the pQCDonset scale, because $\rho^{(2 \delta)}(\sigma)$ for $\sigma>M_{0}^{2}$ is taken equal to the discontinuity function $\rho^{(\mathrm{pt})}(\sigma)$ of the underlying pQCD coupling $a_{s}\left(Q^{2}\right)$. The exact value of $M_{0}^{2}$ depends on the chosen value for $c_{2}$ as the scheme-dependent parameter (for more details, see Table 2 in Ref. [8]).

In $2 \delta \mathrm{anQCD}$, in the low- $\sigma$ regime, i.e., $0<\sigma<M_{0}^{2}$, the behavior of the spectral function $\rho(\sigma) \equiv \operatorname{Im} \mathcal{A}_{1}\left(Q^{2}=-\sigma-\right.$ $i \epsilon)$ is controlled by two positive delta functions. The coupling $\mathcal{A}_{1}\left(Q^{2}\right)$ is presented by a dispersion integral which, in addition to the two delta functions, contains as well the $\rho^{(\mathrm{pt})}(\sigma)$ function. The unknown parameters of the delta functions, and the $M_{0}$ scale, are determined by two requirements $[7,8,30]$. The first one is to effectively match the model with the pQCD at large $\left|Q^{2}\right|>\Lambda^{2}$. Second, the model should reproduce the reported experimental rate for the $\tau$ lepton semihadronic nonstrange $V+A$ decay ratio, i.e., $r_{\tau}^{(D=0)} \approx 0.203$.

The physical spacelike QCD quantities $D\left(Q^{2}\right)$, to be evaluated in anQCD with $\mathcal{A}_{1}$, are in practice, in general, represented in pQCD as a truncated perturbations series of Eq. (9), and using the renormalization scale $\mu^{2}=$ $\kappa Q^{2}(0<\kappa \lesssim 1)$ 


$$
\begin{aligned}
D\left(Q^{2}\right)^{[N]}= & a\left(\kappa Q^{2}\right)^{\nu_{0}}+d_{1}(\kappa) a\left(\kappa Q^{2}\right)^{\nu_{0}+1}+\cdots \\
& +d_{N-1}(\kappa) a\left(\kappa Q^{2}\right)^{\nu_{0}+N-1} .
\end{aligned}
$$

We point out that in anQCD the simple replacement $a\left(\kappa Q^{2}\right)^{\nu_{0}+m} \mapsto \mathcal{A}_{1}\left(\kappa Q^{2}\right)^{\nu_{0}+m}$ in Eq. (11) is not applicable, since it makes the perturbation series diverge rapidly by increasing the power index $N$ (see [31]), and the nonperturbative contributions generated in this way become erratic. Therefore, the formalism introduced in Refs. $[25,26]$ for the case of integer $\nu_{0}$ and the formalism in Ref. [30] for the case of general real $\nu_{0}$ are needed to overcome the difficulty, in any anQCD. Consequently, the following replacement takes place in Eq. (11) in anQCD:

$$
a\left(\kappa Q^{2}\right)^{\nu_{0}+m} \mapsto \mathcal{A}_{\nu_{0}+m}\left(\kappa Q^{2}\right) \quad\left[\neq \mathcal{A}\left(\kappa Q^{2}\right)^{\nu_{0}+m}\right] .
$$

The holomorphic power analog $\mathcal{A}_{\nu_{0}+m}\left(Q^{2}\right)$ can be obtained from $\mathcal{A}_{1}\left(Q^{2}\right)$ by the following construction. First, one needs the logarithmic derivatives of $\mathcal{A}_{1}\left(Q^{2}\right)$, which are given by [30]

$$
\tilde{\mathcal{A}}_{n+1}\left(Q^{2}\right) \equiv \frac{(-1)^{n}}{\beta_{0}^{n} n !}\left(\frac{\partial}{\partial \ln Q^{2}}\right)^{n} \mathcal{A}_{1}\left(Q^{2}\right) \quad(n=0,1,2, \ldots),
$$

where $\beta_{0}=\left(11-2 N_{f} / 3\right) / 4$ and it is obvious that $\tilde{\mathcal{A}}_{1} \equiv \mathcal{A}_{1}$.

Considering anQCD coupling $\mathcal{A}_{1}$, Eq. (13) can be written in terms of the discontinuity function $\rho(\sigma) \equiv$ $\operatorname{Im} \mathcal{A}_{1}(-\sigma-i \epsilon)$, where the Cauchy theorem is used along the cut line. Therefore, Eq. (13) can be written as follows $[25,26,30]$ :

$\tilde{\mathcal{A}}_{n+1}\left(Q^{2}\right)=\frac{1}{\pi} \frac{(-1)}{\beta_{0}^{n} \Gamma(n+1)} \int_{0}^{\infty} \frac{d \sigma}{\sigma} \rho(\sigma) \mathrm{Li}_{-n}\left(-\sigma / Q^{2}\right)$.

Here $\Gamma(n)$ is denoting the usual Gamma function, and $\mathrm{Li}_{-n}$ is defined below in the general case by Eq. (17).

The generalization to the noninteger $n \mapsto \nu$ is given by [30]

$$
\begin{aligned}
\tilde{\mathcal{A}}_{\nu+1}\left(Q^{2}\right)= & \frac{1}{\pi} \frac{(-1)}{\beta_{0}^{\nu} \Gamma(\nu+1)} \int_{0}^{\infty} \frac{d \sigma}{\sigma} \rho(\sigma) \mathrm{Li}_{-\nu}\left(-\frac{\sigma}{Q^{2}}\right) \\
& \times(-1<\nu) .
\end{aligned}
$$

Instead of $\rho$, the above quantity can be expressed in terms of $\mathcal{A}_{1}\left(\equiv \tilde{\mathcal{A}}_{1}\right)[30]$ :

$$
\tilde{\mathcal{A}}_{\delta+m}\left(Q^{2}\right)=K_{\delta, m}\left(\frac{d}{d \ln Q^{2}}\right)^{m} \int_{0}^{1} \frac{d \xi}{\xi} \mathcal{A}_{1}\left(Q^{2} / \xi\right) \ln ^{-\delta}\left(\frac{1}{\xi}\right) .
$$

Here $K_{\delta, m}=(-1)^{m} \beta_{0}^{-\delta-m+1} /[\Gamma(\delta+m) \Gamma(1-\delta)]$, in which $m=0,1,2, \ldots$ and $0 \leq \delta<1$. Equation (16) originates from Eq. (15), using the following expression for the $\mathrm{Li}_{-\nu}(z)$ function [32]:

$$
\begin{aligned}
\mathrm{Li}_{-n-\delta}(z)= & \left(\frac{d}{d \ln z}\right)^{n+1}\left[\frac{z}{\Gamma(1-\delta)} \int_{0}^{1} \frac{d \xi}{1-z \xi} \ln ^{-\delta}\left(\frac{1}{\xi}\right)\right] \\
& (n=-1,0,1, \ldots ; 0<\delta<1) .
\end{aligned}
$$

Combining various generalized logarithmic derivatives, the analytic analogs $\mathcal{A}_{\nu}$ of powers $a^{\nu}$ are obtained [30]:

$$
\mathcal{A}_{\nu}=\tilde{\mathcal{A}}_{\nu}+\sum_{m \geq 1} \tilde{k}_{m}(\nu) \tilde{\mathcal{A}}_{\nu+m}
$$

where the coefficients $\tilde{k}_{m}(\nu)$ were obtained in Ref. [30] for general $\nu$. It can be shown that this approach, in the specific case of APT $\left[\rho(\sigma)=\rho^{(\mathrm{pt})}(\sigma) ; \sigma \geq 0\right]$, gives the same result as the expression $\mathcal{A}_{\nu}^{\text {(FAPT) }}\left(Q^{2}\right)$ in Eq. (1). However, the approach in Eqs. (15)-(18) is applicable in any anQCD. We recall that in the $2 \delta \mathrm{anQCD}$ model at high momenta, $\sigma \geq M_{0}^{2}$, the discontinuity function $\rho(\sigma) \equiv \operatorname{Im} \mathcal{A}_{1}(-\sigma-i \epsilon)$ is given by $\rho^{(\mathrm{pt})}(\sigma)[\equiv \operatorname{Im} a(-\sigma-i \epsilon)]$. In the lowmomentum region, it is parametrized by two delta functions, such that $[7,8]$

$$
\begin{aligned}
\rho^{(2 \delta)}(\sigma)= & \pi F_{1}^{2} \delta\left(\sigma-M_{1}^{2}\right)+\pi F_{2}^{2} \delta\left(\sigma-M_{2}^{2}\right) \\
& +\Theta\left(\sigma-M_{0}^{2}\right) \rho^{(\mathrm{pt})}(\sigma),
\end{aligned}
$$

where $\Theta$ is the step (Heaviside) function. Therefore, the $2 \delta$ anQCD coupling is represented by

$$
\begin{aligned}
\tilde{\mathcal{A}}_{\nu}^{(2 \delta)}\left(Q^{2}\right)= & \frac{(-1)}{\beta_{0}^{\nu} \Gamma(\nu+1)}\left\{\sum_{j=1}^{2} \frac{F_{j}^{2}}{M_{j}^{2}} \operatorname{Li}_{-\nu}\left(-\frac{M_{j}^{2}}{Q^{2}}\right)\right. \\
& \left.+\frac{1}{\pi} \int_{M_{0}^{2}}^{\infty} \frac{d \sigma}{\sigma} \operatorname{Im} a(-\sigma-i \epsilon) \operatorname{Li}_{-\nu}\left(-\frac{\sigma}{Q^{2}}\right)\right\} .
\end{aligned}
$$

In Eq. (20), the parameters $F_{j}^{2}$ and $M_{j}(j=1,2)$ are determined by the requirement that the deviation from the conventional pQCD result at high $Q^{2}>\Lambda^{2}$ is such that $\mathcal{A}_{\nu}^{(2 \delta)}\left(Q^{2}\right)-a\left(Q^{2}\right)^{\nu} \sim\left(\Lambda^{2} / Q^{2}\right)^{5}$, i.e., essentially zero [in (F)APT, this deviation is $\left.\sim\left(\Lambda^{2} / Q^{2}\right)^{1}\right]$. These are, in fact, four requirements. The pQCD-onset scale $M_{0}$ is fixed by the condition that the model should reproduce the measured semihadronic decay ratio $r_{\tau} \approx 0.203$ for the strangeless and massless $V+A$ tau lepton [7,8,33].

In the $2 \delta$ anQCD model, the coupling $a$ of the underlying $\mathrm{pQCD}$ is chosen for convenience in terms of the Lambert$W$ function (which Mathematica can evaluate without problems) such that 


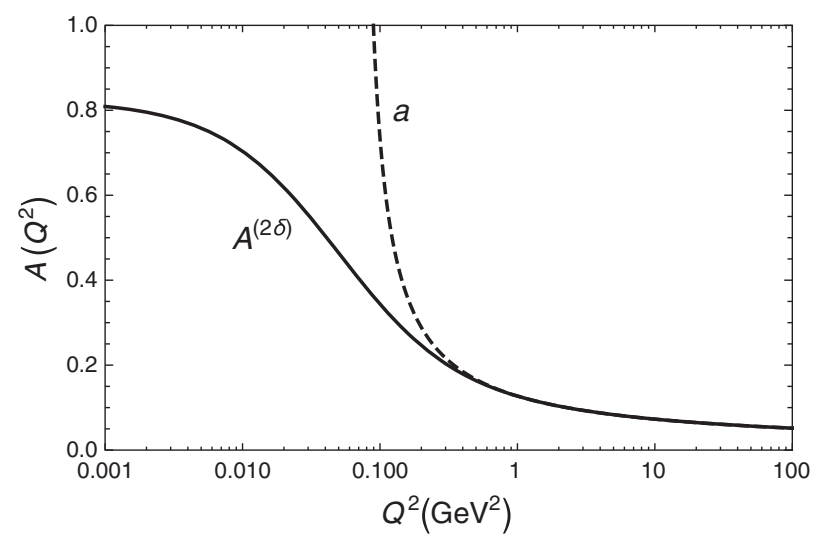

FIG. 1. The coupling $\mathcal{A}_{1}^{(2 \delta)}$ in $2 \delta$ anQCD, with $N_{f}=3$, as a function of $Q^{2}$. The dashed line represents the underlying pQCD coupling Eq. (21), with $c_{2}=-4.9$.

$$
a\left(Q^{2}\right)=-\frac{1}{c_{1}} \frac{1}{\left[1-c_{2} / c_{1}^{2}+W_{\mp 1}\left(z_{ \pm}\right)\right]} .
$$

As defined before, in this equation $c_{1}=\beta_{1} / \beta_{0}$, $c_{2}=\beta_{2} / \beta_{0}$, and $Q^{2}=\left|Q^{2}\right| \mathrm{e}^{i \phi}$, where $\phi$ is the required argument in which one can follow the calculations in the complex $Q^{2}$ plane. The two universal beta coefficients are $\beta_{0}=\left(11-2 N_{f} / 3\right) / 4$ and $\beta_{1}=\left(102-38 N_{f} / 3\right) / 16$. The upper and lower sign refer to $\phi \geq 0$ and $\phi<0$, respectively, and the $z$ variable is

$z_{ \pm}=\left(c_{1} \mathrm{e}\right)^{-1}\left(\left|Q^{2}\right| / \Lambda_{L}^{2}\right)^{-\beta_{0} / c_{1}} \exp \left[i\left( \pm \pi-\beta_{0} \phi / c_{1}\right)\right]$.

The coupling (21) is in a renormalization scheme called the Lambert scheme. In these schemes, $c_{n}\left(\equiv \beta_{n} / \beta_{0}\right)=c_{2}^{n-1} /$ $c_{1}^{n-2}(n=3,4, \ldots)$; i.e., all the higher $\beta_{n}$ scheme parameters $(n=3,4, \ldots)$ are fixed by $c_{2}$ [8]. The acceptable values of the scheme parameter $c_{2}$ can be in the interval $-5.6<c_{2}<-2$, with $c_{2}=-4.9$ as the preferred (and chosen) value [8]. The Lambert scale $\Lambda_{L}=0.255 \mathrm{GeV}$ is determined in such a way that it corresponds in the $\overline{\mathrm{MS}}$ scheme at $\mu^{2}=k, Q^{2}=M_{Z}^{2}$ [with $N_{f}=5$ and $k=1$; see the text below Eq. (51)] to the value $\alpha_{s}\left(M_{Z}^{2} ; \overline{\mathrm{MS}}\right)=0.1184$. The scaling in the pQCD coupling (21) corresponds to the $\overline{\mathrm{MS}}$ scaling, i.e., $a\left(Q^{2}\right)-a^{\overline{\mathrm{MS}}}\left(Q^{2}\right) \sim a^{3}$.

In Fig. 1 , the $2 \delta$ anQCD running strong coupling is plotted as a function of $Q^{2}$ and compared with the coupling of the underlying pQCD. As can be seen, the $2 \delta$ anQCD coupling $\mathcal{A}_{1}$ has a finite value when $Q^{2} \rightarrow 0$, while the coupling $a$ from the underlying pQCD increases rapidly and goes to infinity near the Landau branching point $Q^{2}=\Lambda^{2}$.

Keeping in mind Eq. (18), any QCD observable can be calculated within $2 \delta$ anQCD (or any anQCD). In the following sections, we employ this model to analyze the polarized nucleon structure function and the related Bjorken sum rule.

\section{NLO EVOLUTION OF POLARIZED PARTON DENSITIES, RATIONAL APPROXIMATION, AND JACOBI POLYNOMIALS}

We evaluate here the polarized nucleon structure function. The precision depends on the method to calculate the structure function. It is therefore necessary to characterize the employed method. One of the reliable methods is the use of the Jacobi polynomials, which leads to sufficient precision in the evolution of the function with $Q^{2}$. Alternatively, using the inverse Mellin transformation, the result in Bjorken $x$ space can be directly obtained from the evolved moments of the structure functions in $n$ space.

Before employing the Jacobi method, we present some technical details about the evolution of the polarized parton densities in the moment $n$ space. In this context, an approximate method (rational approximation) is introduced which yields required expansions for the considered moments. For this purpose, we need $\Delta P_{\mathrm{NS}}^{(0)}(n)$, which is the $n$th moment of the nonsinglet splitting function at the leading order [34]:

$$
\Delta P_{\mathrm{NS}}^{(0)}(n)=\frac{4}{3}\left[\frac{3}{2}+\frac{1}{n(n+1)}-2 S_{1}(n)\right] .
$$

In this equation, $S_{1}(n) \equiv \sum_{j=1}^{n} 1 / j=\psi(n+1)+\gamma_{E}$, where $\psi(n) \equiv \Gamma^{\prime}(n) / \Gamma(n)$ and $\gamma_{E}=0.577216$ is the Euler-Mascheroni constant.

As we specify later on, our calculations throughout the whole manuscript are based on using the $2 \delta$ anQCD model alongside the underlying $\mathrm{pQCD}$ as represented by the (pQCD) coupling Eq. (21). They are both in the specific Lambert $\left(c_{2}=-4.9\right)$ scheme, but in the rest of this section we use terms and phrases which are valid in general schemes. In the next section, which contains the main results for the nucleon structure function $g_{1}$, the required expressions are used in the Lambert scheme, but here a general discussion is followed. In this regard, we resort to a solution of the renormalization group equation for the pQCD coupling in NLO approximation that is given in Ref. [35]. Then the solution of the NLO evolution equation for the moments of the structure function leads to [36]

$$
\begin{aligned}
& \Delta M_{\mathrm{NS}}\left(n, Q^{2}\right) \\
& =\frac{1+\Delta C_{\mathrm{NS}}^{(1)}(n) a_{s}\left(Q^{2}\right)}{1+\Delta C_{\mathrm{NS}}^{(1)}(n) a_{s}\left(Q_{0}^{2}\right)}\left(\frac{1+\left(b_{1} / b_{0}\right) a_{s}\left(Q^{2}\right)}{1+\left(b_{1} / b_{0}\right) a_{s}\left(Q_{0}^{2}\right)}\right)^{\Delta p(n)} \\
& \quad \times\left[\frac{a_{s}\left(Q^{2}\right)}{a_{s}\left(Q_{0}^{2}\right)}\right]^{\Delta d_{\mathrm{NS}}(n)} \Delta M_{\mathrm{NS}}\left(n, Q_{0}^{2}\right) .
\end{aligned}
$$

In the above equation, the Wilson coefficient $\Delta C_{\mathrm{NS}}^{(1)}$ is given by [34] 


$$
\begin{aligned}
\Delta C_{\mathrm{NS}}^{(1)}= & \frac{4}{3}\left[-S_{2}(n)+\left(S_{1}(n)\right)^{2}+\left(\frac{3}{2}-\frac{1}{n(n+1)}\right)\right. \\
& \left.\times S_{1}(n)+\frac{1}{n^{2}}+\frac{1}{2 n}+\frac{1}{n+1}-\frac{9}{2}\right],
\end{aligned}
$$

where $S_{2}(n) \equiv \sum_{j=1}^{n} 1 / j^{2}=\pi^{2} / 6-\psi^{\prime}(n+1)$ in which $\psi^{\prime}(n)=(d / d n)^{2} \ln \Gamma(n)$.

In Eq. (24), $\Delta M_{\mathrm{NS}}\left(n, Q_{0}^{2}\right)$ can be obtained from the moment of the polarized parton densities, $\Delta f_{\mathrm{NS}}\left(n, Q_{0}^{2}\right)$, as follows:

$$
\Delta M_{\mathrm{NS}}\left(n, Q_{0}^{2}\right)=\left(1+\Delta C_{\mathrm{NS}}^{(1)}(n) a_{s}\left(Q_{0}^{2}\right)\right) \Delta f_{\mathrm{NS}}\left(n, Q_{0}^{2}\right)
$$

$\Delta f_{\mathrm{NS}}\left(n, Q_{0}^{2}\right)$, the moment of the parton densities at the initial scale $Q_{0}^{2}$, will be discussed later on in this section. The functions $\Delta d_{\mathrm{NS}}(n)$ and $\Delta p(n)$ in Eq. (24) are the moments of the splitting functions and are given, respectively, by [36]

$$
\begin{gathered}
\Delta d_{\mathrm{NS}}(n)=-2 \Delta P_{\mathrm{NS}}^{(0)}(n) / b_{0}, \\
\Delta p(n)=-4\left(\frac{\Delta P_{\mathrm{NS}}^{(1)}(n)}{b_{1}}-\frac{\Delta P_{\mathrm{NS}}^{(0)}(n)}{2 b_{0}}\right) .
\end{gathered}
$$

Here, the explicit expression for $\Delta P_{\mathrm{NS} \pm}^{(1)}$ is required which is presented in the Appendix.

One of the methods to obtain a solution for the moments of the structure function in Eq. (24) is to use the rational approximation, which is indispensable when we employ the analytic perturbation theory. On this basis, the following factor in Eq. (24):

$$
\left(\frac{1+\left(b_{1} / b_{0}\right) a_{s}\left(Q^{2}\right)}{1+\left(b_{1} / b_{0}\right) a_{s}\left(Q_{0}^{2}\right)}\right)^{\Delta p(n)}
$$

which is denoted by $\Delta m\left(n, Q^{2}\right)$, has the following expansions up to $\mathcal{O}(a)$ and $\mathcal{O}\left(a^{2}\right)$ accuracy, respectively [36]:

$$
\begin{aligned}
\Delta m_{\mathrm{pQCD}}^{(1)}\left(n, Q^{2}\right) & \simeq \frac{1+\left(b_{1} / b_{0}\right) \Delta p(n) a_{s}\left(Q^{2}\right)}{1+\left(b_{1} / b_{0}\right) \Delta p(n) a_{s}\left(Q_{0}^{2}\right)} \\
\Delta m_{\mathrm{pQCD}}^{(2)}\left(n, Q^{2}\right) & \simeq \frac{1+\left(b_{1} / b_{0}\right) \Delta p(n) a_{s}\left(Q^{2}\right)+\left(b_{1}^{2} / 2 b_{0}^{2}\right) \Delta p(n)(\Delta p(n)-1) a_{s}^{2}\left(Q^{2}\right)}{1+\left(b_{1} / b_{0}\right) \Delta p(n) a_{s}\left(Q_{0}^{2}\right)+\left(b_{1}^{2} / 2 b_{0}^{2}\right) \Delta p(n)(\Delta p(n)-1) a_{s}^{2}\left(Q_{0}^{2}\right)}
\end{aligned}
$$

The polarized splitting function $\Delta p(n)$ in Eq. (30) is obtained by Eq. (28).

To construct the moments of structure functions, one needs the polarized parton densities at initial $Q_{0}^{2}$ as the input densities. We take these densities or parton distribution functions from the data-based parameterization model like KATAO at $Q_{0}^{2}=4 \mathrm{GeV}^{2}$ such that [37]

$x \Delta u_{v}\left(x, Q_{0}^{2}\right)=\mathcal{N}_{u_{v}} \eta_{u_{v}} x^{\alpha_{u_{v}}}(1-x)^{\beta_{u_{v}}}\left(1+\gamma_{u_{v}} x\right)$,
$x \Delta d_{v}\left(x, Q_{0}^{2}\right)=\mathcal{N}_{d_{v}} \eta_{d_{v}} x^{\alpha_{d_{v}}}(1-x)^{\beta_{d_{v}}}\left(1+\gamma_{d_{v}} x\right)$.

One of the advantages of the value $Q_{0}^{2}=4 \mathrm{GeV}^{2}$ here is that the $2 \delta$ anQCD coupling at this scale practically coincides with the underlying pQCD coupling (21). The numerical values for $\eta_{q_{v}}$ and $\alpha_{q_{v}}, \beta_{q_{v}}$, and $\gamma_{q_{v}}$ parameters are listed in Table I. The normalization constants $\mathcal{N}_{q_{v}}$,

TABLE I. Numerical values for the first moment parameters of $\Delta u_{v}$ and $\Delta d_{v}$ and their statistical errors at the input scale $Q_{0}^{2}=4 \mathrm{GeV}^{2}$ in the NLO approximation, based on the KATAO parameterization model [37].

\begin{tabular}{cccccc}
\hline \hline \multirow{4}{*}{$\Delta u_{v}$} & $\eta_{u_{v}}$ & 0.928 (fixed) & & $\eta_{d_{v}}$ & -0.342 (fixed) \\
& $\alpha_{u_{v}}$ & $0.535 \pm 0.022$ & $\Delta d_{v}$ & $\alpha_{d_{v}}$ & $0.530 \pm 0.067$ \\
& $\beta_{u_{v}}$ & $3.222 \pm 0.085$ & & $\beta_{d_{v}}$ & $3.878 \pm 0.451$ \\
& $\gamma_{u_{v}}$ & 8.180 (fixed) & & $\gamma_{d_{v}}$ & 4.789 (fixed) \\
\hline \hline
\end{tabular}

$$
\frac{1}{\mathcal{N}_{q_{v}}}=\left(1+\gamma_{q_{v}} \frac{\alpha_{q_{v}}}{\alpha_{q_{v}}+\beta_{q_{v}}+1}\right) B\left(\alpha_{q_{v}}, \beta_{q_{v}}+1\right),
$$

are chosen such that $\eta_{q_{v}}$ is considered as the first moment of $x \Delta q_{v}\left(x, Q_{0}^{2}\right)$, i.e., $\eta_{q_{v}}=\int_{0}^{1} d x \Delta q_{v}\left(x, Q_{0}^{2}\right)$. The $B(a, b)$ in Eq. (32) is the Euler beta function. Details of computations to obtain numerical values for the first moments of $\Delta u_{v}$ and $\Delta d_{v}$ are presented in the following subsection. At the NLO approximation, the results of calculations are scheme independent ( $c_{2}$ independent), and we can use the Lambert scheme.

\section{A. First moments of $\Delta u_{v}$ and $\Delta d_{v}$}

The parameters $\eta_{u_{v}}$ and $\eta_{d_{v}}$ are the first moments of the polarized valence quark densities $\Delta u_{v}$ and $\Delta d_{v}$, respectively. These moments can be related to $F$ and $D$ quantities [38] as measured in neutron and hyperon $\beta$ decays

$$
\begin{aligned}
& a_{3}=\int_{0}^{1} d x \Delta q_{3}=\eta_{u_{v}}-\eta_{d_{v}}=F+D, \\
& a_{8}=\int_{0}^{1} d x \Delta q_{8}=\eta_{u_{v}}+\eta_{d_{v}}=3 F-D .
\end{aligned}
$$

In these equations, $a_{3}$ and $a_{8}$ are the nonsinglet combinations of the first moments which are constructed from the polarized parton densities such that 


$$
\begin{gathered}
\Delta q_{3}=(\Delta u+\Delta \bar{u})-(\Delta d+\Delta \bar{d}), \\
\Delta q_{8}=(\Delta u+\Delta \bar{u})+(\Delta d+\Delta \bar{d})-2(\Delta s+\Delta \bar{s}) .
\end{gathered}
$$

Doing a reanalysis for $F$ and $D$ with updated $\beta$-decay constants, one obtains $F=0.464 \pm 0.008$ and $D=$ $0.806 \pm 0.008$ [38]. Based on Eq. (33) and considering the experimental values for $F$ and $D$, the following numerical values are obtained for the first moments of the polarized valence densities:

$$
\begin{aligned}
& \eta_{u_{v}}=+0.928 \pm 0.014, \\
& \eta_{d_{v}}=-0.342 \pm 0.018 .
\end{aligned}
$$

Full results for the moments of the nucleon polarized structure functions at the NLO approximation are now accessible. It is therefore necessary to obtain the structure functions in Bjorken- $x$ space. This can be done using the Jacobi polynomial method, which we summarize in the next subsection.

\section{B. The Jacobi polynomial method}

Based on the Jacobi polynomial method, the polarized structure function $x g_{1}^{(\mathrm{NS})}\left(x, Q^{2}\right)=x g_{1}^{p}\left(x, Q^{2}\right)-x g_{1}^{n}\left(x, Q^{2}\right)$ can be expanded as follows [37,39-42]:

$$
x g_{1}^{\mathrm{NS}}\left(x, Q^{2}\right)=x^{\beta}(1-x)^{\alpha} \sum_{n=0}^{N_{\max }} a_{n}\left(Q^{2}\right) \Theta_{n}^{\alpha, \beta}(x) .
$$

In the above equation, $\Theta_{n}^{\alpha, \beta}(x)$ are Jacobi polynomials of order $n$, and $N_{\max }$ is the maximum order of expansion. Jacobi polynomials provide a method to separate the main part of the $x$ dependence of the structure function into the weight function $x^{\beta}(1-x)^{\alpha}$, while the $Q^{2}$ dependence is contained in the Jacobi moments $a_{n}\left(Q^{2}\right)$ [43].

Jacobi polynomials fulfill an orthogonality relation

$$
\int_{0}^{1} d x x^{\beta}(1-x)^{\alpha} \Theta_{k}^{\alpha, \beta}(x) \Theta_{l}^{\alpha, \beta}(x)=\delta_{k, l} .
$$

Using this, Eq. (39) can be inverted to yield the Jacobi moments $a_{n}\left(Q^{2}\right)$ :

$$
\begin{aligned}
a_{n}\left(Q^{2}\right) & =\int_{0}^{1} d x x g_{1}\left(x, Q^{2}\right) \Theta_{n}^{\alpha, \beta}(x) \\
& =\sum_{j=0}^{n} c_{j}^{(n)}(\alpha, \beta) \Delta M\left[x g_{1}^{\mathrm{NS}}, j+2\right] .
\end{aligned}
$$

To derive the last line in the above equation, it is needed to substitute Eq. (39) for $x g_{1}^{\mathrm{NS}}\left(x, Q^{2}\right)$ into the first line of Eq. (41) and to use the Mellin transform

$$
\Delta M\left[x g_{1}^{\mathrm{NS}}, N\right] \equiv \int_{0}^{1} d x x^{N-2} x g_{1}^{\mathrm{NS}}\left(x, Q^{2}\right)
$$

The polarized structure function $x g_{1}^{\mathrm{NS}}\left(x, Q^{2}\right)$ can now be related to Mellin moments as follows [37]:

$$
\begin{aligned}
x g_{1}^{\mathrm{NS}}\left(x, Q^{2}\right)= & x^{\beta}(1-x)^{\alpha} \sum_{n=0}^{N_{\max }} \Theta_{n}^{\alpha, \beta}(x) \\
& \times \sum_{j=0}^{n} c_{j}^{(n)}(\alpha, \beta) \Delta M\left[x g_{1}^{\mathrm{NS}}, j+2\right] .
\end{aligned}
$$

It is required to choose the set $\left\{N_{\max }, \alpha, \beta\right\}$ such that an optimal convergence of the series is achieved. This convergence should contain the whole kinematic region and cover the related experimental data. An improvement is achieved for $\alpha=3.0$, and $\beta=0.5$ while $N_{\max }$ varies between 7 and 9 [42].

As an alternative to the above method, the structure function $x g_{1}^{\mathrm{NS}}\left(x, Q^{2}\right)$ may be obtained by using the inverse Mellin transform for the moments of the structure function. On this basis, one obtains for $x g_{1}^{\mathrm{NS}}\left(x, Q^{2}\right)$ the following expression in which a convenient path of integration is chosen:

$$
\begin{gathered}
x g_{1}^{\mathrm{NS}}\left(x, Q^{2}\right)=\frac{1}{\pi} \int_{0}^{5+[10 / \ln (1 / x)]} d z \operatorname{Im}\left[e^{i \phi} x^{1-c-z \exp (i \phi)}\right. \\
\left.\Delta M_{\mathrm{NS}}\left(N=c+z e^{i \phi}\right)\right] .
\end{gathered}
$$

In this integration, it is assumed $c=1.9$ and $\phi=\frac{3 \pi}{4}$ [44].

Now we are able to construct the polarized structure function in the $2 \delta$ anQCD model, based on the Jacobi polynomials which is considered in the following section.

\section{EXTRACTING THE POLARIZED STRUCTURE FUNCTION, USING THE $2 \delta$ anQCD MODEL}

Here we are going to employ the $2 \delta$ anQCD model to construct the polarized nucleon structure function. This model, like the underlying pQCD based on Eq. (21) as the coupling constant of series expansions, is considered in the Lambert scheme.

In the anQCD approach, we replace the power $a_{s}^{\nu} \equiv$ $a^{\nu} / 4^{\nu}$ of the conventional pQCD by the analytic coupling $\mathcal{A}_{\nu} / 4^{\nu}$. Note that $\mathcal{A}_{\nu}\left(Q^{2}\right)$ is the analog (image) of $a\left(Q^{2}\right)^{\nu} \equiv$ $\left(\alpha_{s}\left(Q^{2}\right) / \pi\right)^{\nu}$, and $a_{s}\left(Q^{2}\right)=a\left(Q^{2}\right) / 4$. In this way, the moments $\Delta \mathcal{M}_{\mathrm{NS}}$ as the analytic images of $\Delta M_{\mathrm{NS}}$ are obtained. We recall that $\nu$ in the analytic coupling $\mathcal{A}_{\nu}$ is an expansion order index rather than a power index. For simplicity, we will denote in this section

$$
A_{s, \nu}\left(Q^{2}\right) \equiv \frac{\mathcal{A}_{\nu}\left(Q^{2}\right)}{4^{\nu}}
$$

i.e., $A_{s, \nu}$ is the anQCD image (analog) of $a_{s}^{\nu}$, while $\mathcal{A}_{\nu}$ is (always) the anQCD image (analog) of $a^{\nu}$. When the first rational approximation in Eq. (30) is employed, the mentioned replacement in Eq. (24) leads to the following result [18]: 
$\Delta \mathcal{M}_{\mathrm{NS}}\left(n, Q^{2}\right)$

$$
\begin{aligned}
= & \frac{A_{s, \Delta d_{\mathrm{NS}}(n)}\left(Q^{2}\right)+\left(\Delta C_{\mathrm{NS}}^{(1)}(n)+\frac{b_{1}}{b_{0}} \Delta p(n)\right) A_{s, \Delta d_{\mathrm{NS}}(n)+1}\left(Q^{2}\right)}{A_{s, \Delta d_{\mathrm{NS}}(n)}\left(Q_{0}^{2}\right)+\left(\Delta C_{\mathrm{NS}}^{(1)}(n)+\frac{b_{1}}{b_{0}} \Delta p(n)\right) A_{s, \Delta d_{\mathrm{NS}}(n)+1}\left(Q_{0}^{2}\right)} \\
& \times \Delta \mathcal{M}_{\mathrm{NS}}\left(n, Q_{0}^{2}\right) .
\end{aligned}
$$

We then use this formalism and evaluate $A_{s, \nu}$ with the help of the Mathematica package that is called anQCD.m as introduced in Ref. [8].

Using the corresponding command for the analytic coupling $\mathcal{A}_{\nu}$ in the $2 \delta \mathrm{anQCD}$ model in the two-loop approximation, we write [8]

$$
A_{s, \nu}(Q 2)=\frac{A 2 d 2 l[3,0, \nu, Q 2,0]}{4^{\nu}} .
$$

Substituting Eq. (47) in Eq. (46), with $Q 2=Q^{2}>0$, leads to the numerical result for the moments of the structure function in the anQCD approach. We note that there are some other commands in Ref. [8] for the anQCD coupling

in $2 \delta$ anQCD model, but the one in Eq. (47) is more appropriate for our numerical purpose with sufficient precision. Namely, $\mathcal{A}_{\nu}\left(Q^{2}\right)=A 2 d 2 l[3,0, \nu, Q 2,0]$, for positive $Q^{2}=Q 2>0$ and with $N_{f}=3$, is constructed as the truncated sum of two terms in Eq. (18), i.e., $\mathcal{A}_{\nu}=\tilde{\mathcal{A}}_{\nu}+\tilde{k}_{1}(\nu) \tilde{\mathcal{A}}_{\nu+1}$, which is sufficient for our NLO analysis.

As more explanation, it should be said that $A 2 d N l\left[N_{f}\right.$, $\left.n, \nu,\left|Q^{2}\right|, \phi\right]$ is representing the $N$-loop analytic $2 \delta$ anQCD coupling $\mathcal{A}_{n+\nu}^{(2 \delta)}\left(Q^{2}, N_{f}\right)$ of fractional power $n+\nu$, where $\nu>-1$ and index $n$ is such that $n=0,1, \ldots, N-1$. Here the active quark flavor $N_{f}$ is fixed. In the Euclidean domain, the energy scale $Q^{2}$ is given by $Q^{2}=$ $\left|Q^{2}\right| \exp (i \phi) \in \mathcal{C} \backslash\left[-M_{\mathrm{thr}}^{2},-\infty\right)$ in which $M_{\mathrm{thr}}^{2}=M_{2}^{2}$ that is applicable for the $\mathrm{N}^{n-1} \mathrm{LO}$ truncation approach [8].

It is evident that the anQCD analog of Eq. (30) is

$$
\Delta m_{\mathrm{anQCD}}^{(1)}\left(n, Q^{2}\right) \simeq \frac{1+\left(b_{1} / b_{0}\right) \Delta p(n) A_{s, 1}\left(Q^{2}\right)}{1+\left(b_{1} / b_{0}\right) \Delta p(n) A_{s, 1}\left(Q_{0}^{2}\right)},
$$

$$
\Delta m_{\mathrm{anQCD}}^{(2)}\left(n, Q^{2}\right) \simeq \frac{1+\left(b_{1} / b_{0}\right) \Delta p(n) A_{s, 1}\left(Q^{2}\right)+\left(b_{1}^{2} / 2 b_{0}^{2}\right) \Delta p(n)(\Delta p(n)-1) A_{s, 2}\left(Q^{2}\right)}{1+\left(b_{1} / b_{0}\right) \Delta p(n) A_{s, 1}\left(Q_{0}^{2}\right)+\left(b_{1}^{2} / 2 b_{0}^{2}\right) \Delta p(n)(\Delta p(n)-1) A_{s, 2}\left(Q_{0}^{2}\right)}
$$

One can construct from Eqs. (30) a combined quantity such that $\Delta m^{(1,2)}=\left|\Delta m^{(1)}-\Delta m^{(2)}\right| / \Delta m^{(1)}$. As a result, we obtain an accuracy better than $1 \%$ for any $n \leq 11$, while the expansion of Jacobi polynomials contains nine terms to yield us a good approximation. This accuracy is obtained for both underlying $\mathrm{pQCD}$ and analytic perturbation theory based on the $2 \delta$ anQCD model. The numerical results for this accuracy at the energy scale $Q^{2} \approx 0.17 \mathrm{GeV}^{2}$ are collected in Table II.

This confirms that the first rational approximation, $\Delta m_{\mathrm{anQCD}}^{(1)}\left(n, Q^{2}\right)$ in Eq. (48), has sufficient precision; i.e., the corresponding Eq. (46) gives us the desired results for the moments of the polarized nucleon structure function in the $2 \delta \mathrm{anQCD}$ approach. The results, using these two approximations, are depicted in Fig. 2, i.e., the polarized structure function $g_{1}^{(\mathrm{NS})}$ as a function of $Q^{2}$ at $x=0.03$. The plots in this figure denoted by $2 \delta \mathrm{anQCD}^{(1)}$ and

TABLE II. The accuracy in percent for the difference of the approximations $\Delta m^{(1,2)}=\frac{\left|\Delta m^{(1)}-\Delta m^{(2)}\right|}{\Delta m^{(1)}}$ in the underlying pQCD, $\Delta m_{\mathrm{pQCD}}^{(1,2)}$; and for anQCD in $2 \delta$ anQCD, $\Delta m_{\mathrm{anQCD}}^{(1,2)}$. The results are presented for $Q^{2} \approx 0.17 \mathrm{GeV}^{2}$.

\begin{tabular}{lccccc}
\hline \hline$n$ & 2 & 4 & 6 & 8 & 10 \\
\hline$\Delta m_{\mathrm{pQCD}}^{(1,2)} \%$ & 1.372 & 0.817 & 0.177 & 0.368 & 0.824 \\
$\Delta m_{\mathrm{anQCD}}^{(1,2)} \%$ & 0.499 & 0.296 & 0.0642 & 0.132 & 0.296 \\
\hline \hline
\end{tabular}

$2 \delta \mathrm{anQCD}^{(2)}$, which are related to the two approximate expansions in Eqs. (48) and (49), are in good agreement with each other and also with the available experimental data. In this figure, some of the data are from Refs. [45-47] which have been collected in Table 1 in Ref. [48]. The data

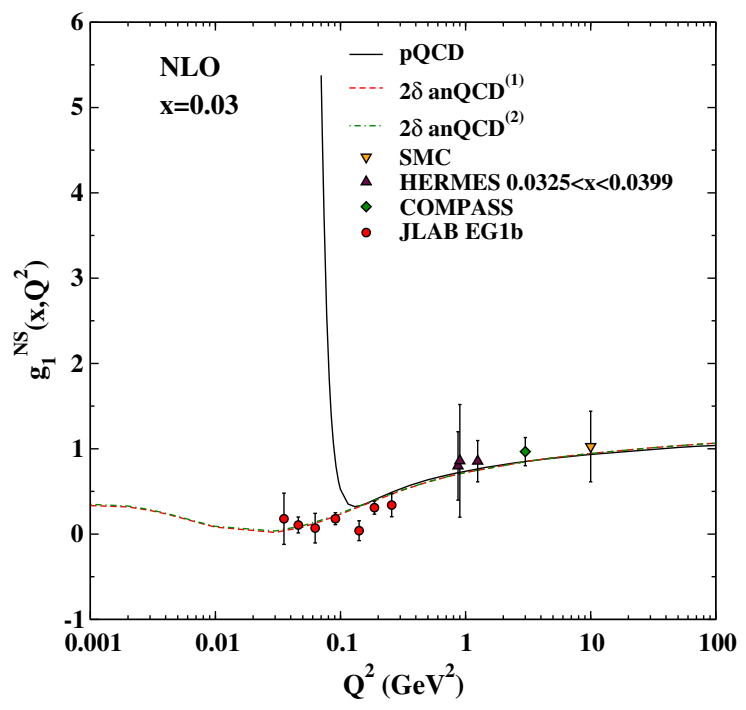

FIG. 2. Nonsinglet structure function $g_{1}^{(\mathrm{NS})}\left(x, Q^{2}\right)$ as a function of $Q^{2}$ at NLO. The dashed lines represent the $2 \delta$ anQCD results, using $\Delta m_{\mathrm{anQCD}}^{(1)}$ and $\Delta m_{\mathrm{anQCD}}^{(2)}$ in Eqs. (48) and (49). The solid line is the underlying $\mathrm{pQCD}$ one. A comparison with the available experimental data $[45-47,49]$ has also been done. 

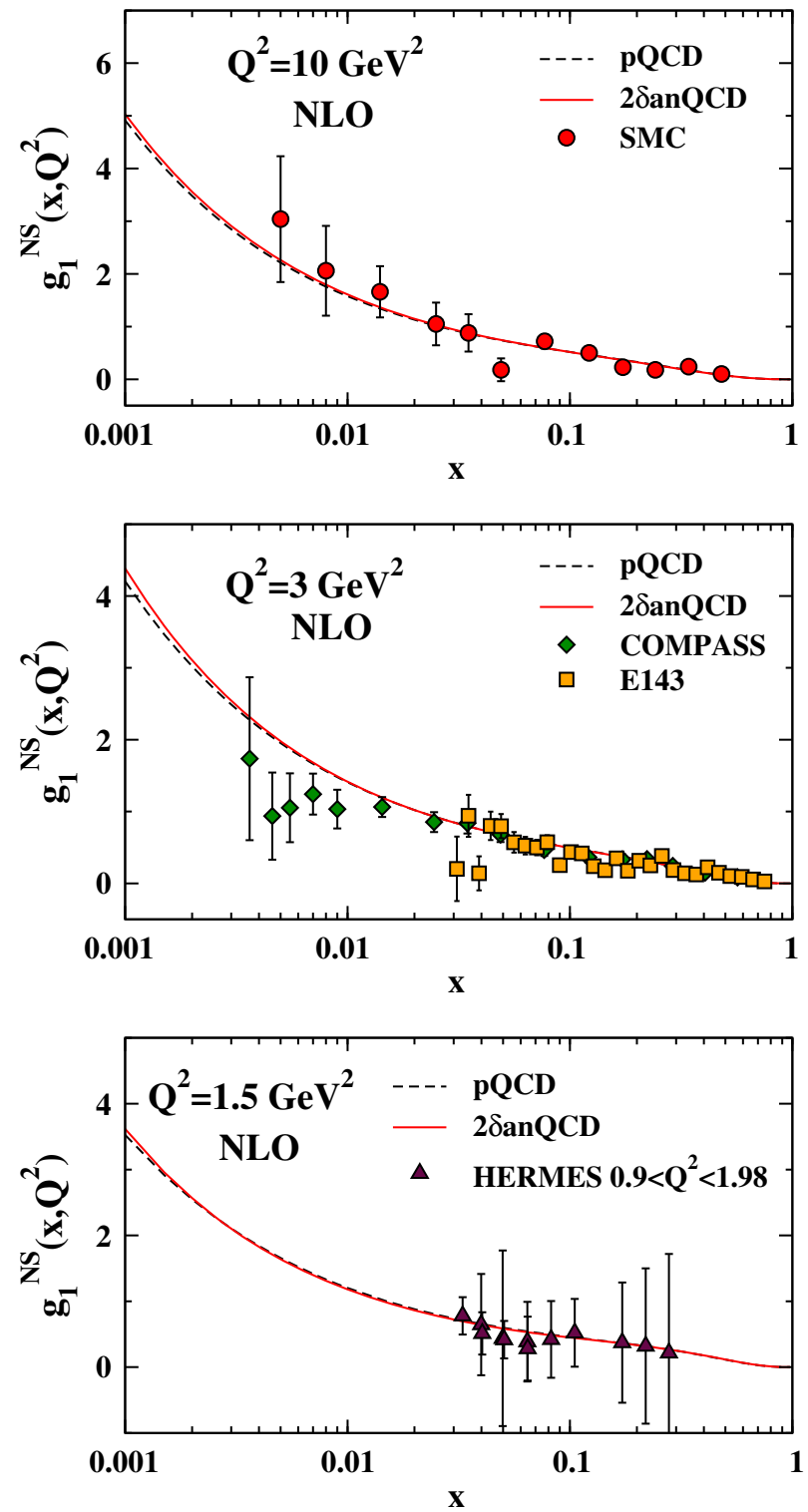

FIG. 3. Nonsinglet structure function $g_{1}^{(\mathrm{NS})}\left(x, Q^{2}\right)$ as a function of $x$ in the NLO approximation. The solid line represents the $2 \delta$ anQCD result, and the dashed line the underlying pQCD one. The available experimental data $[46,47,50,51]$ are included.

at very low-energy scales, i.e., $Q^{2}<0.5 \mathrm{GeV}^{2}$, is from Ref. [49]. The plot for $g_{1}^{(\mathrm{NS})}$ at $x=0.03$ provides an opportunity to compare the available experimental data at low-energy scales with the theoretical prediction of $2 \delta$ anQCD model, where the underlying pQCD does not give an acceptable behavior. This confirms the advantage and applicability of the anQCD approach in comparison to pQCD, especially at the low-energy scales. In Fig. 3, we present the results for $g_{1}^{(\mathrm{NS})}$ as a function of $x$, at $Q^{2}=10$, $3,1.5$, and $0.5 \mathrm{GeV}^{2}$ in $2 \delta$ anQCD and in the underlying pQCD, and compare them with the available experimental data from the SLAC [50], HERMES [51], SMC [46], and COMPASS [47] experimental groups. All these data have
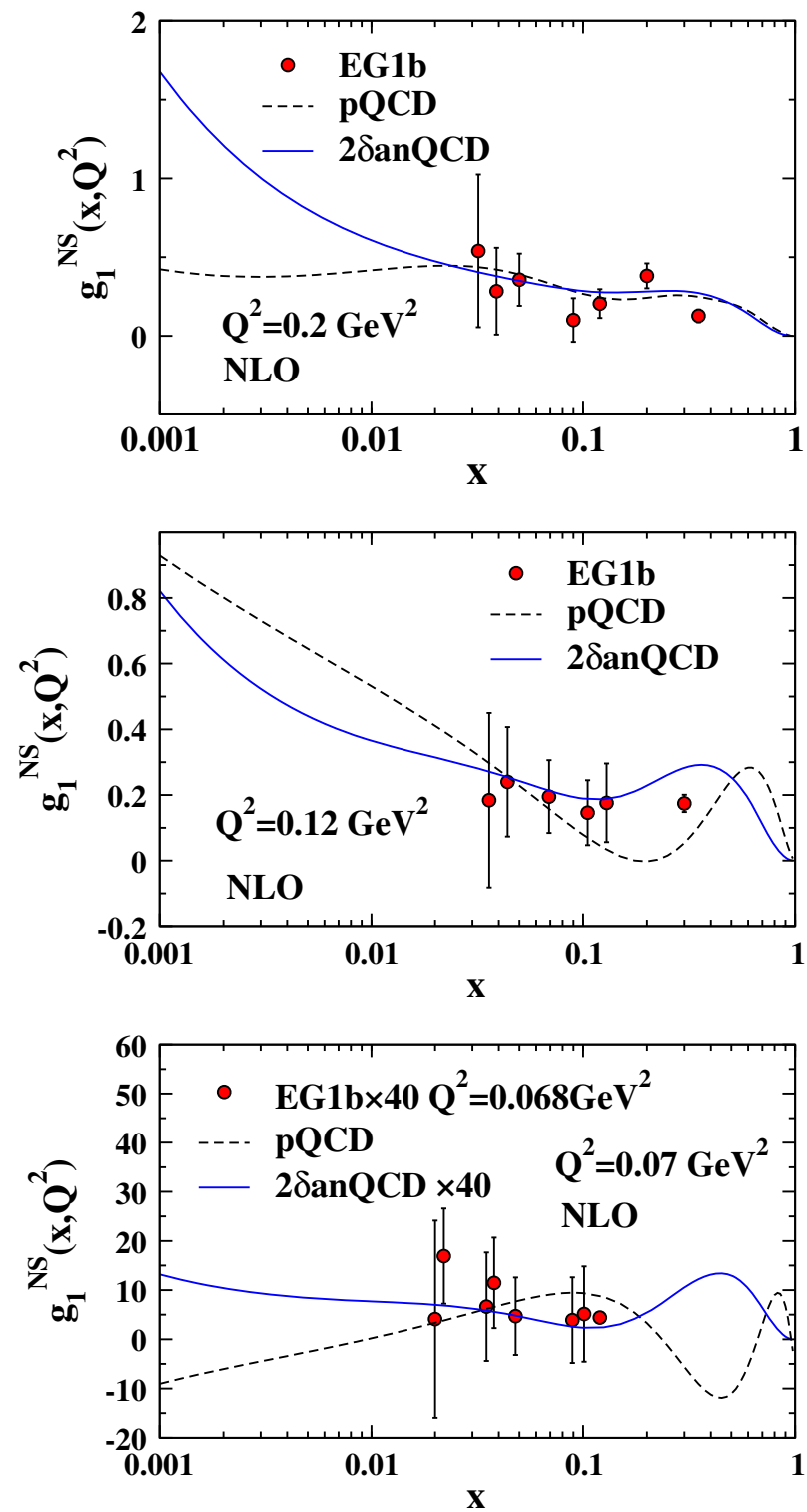

FIG. 4. Nonsinglet structure function $g_{1}^{(\mathrm{NS})}\left(x, Q^{2}\right)$ as a function of $x$ in the NLO approximation at low-energy scales. The solid line with smooth behavior represents the $2 \delta$ anQCD result, and the dashed line the underlying $\mathrm{pQCD}$ one. The available experimental data [49] are included.

been collected in Table 1 in Ref. [48]. As expected, at highand medium-energy scales, there is good agrement between the theoretical predictions and the experimental data. Figure 4 is like Fig. 3 but at low-energy scales $Q^{2}=0.2,0.12$, and $0.07 \mathrm{GeV}^{2}$. By decreasing the energy scales, the difference between the $2 \delta \mathrm{anQCD}$ and the underlying pQCD results increases. At $Q^{2}=0.07 \mathrm{GeV}^{2}$, the underlying pQCD result for $x \geq 0.1$ as a function of increasing $x$ grows rapidly and also oscillates. As a consequence, we multiplied there the $2 \delta \mathrm{anQCD}$ results and the experimental results by a factor of 40 in order to facilitate the comparison with the pQCD results. Figure 4 
indicates that the $g_{1}^{(\mathrm{NS})}$ structure function involves smooth behavior at very low-energy scales, using the $2 \delta \mathrm{anQCD}$ model. This can be considered as an advantage of this model in comparison with underlying pQCD.

There are not enough experimental data for $g_{1}^{(\mathrm{NS})}$ at the very low-energy scales $Q^{2}$. But there are individual experimental data for the polarized proton and neutron structure functions $g_{1}^{p}$ and $g_{1}^{n}$, respectively. Unfortunately, most of them are binned very differently with respect to the $x$-Bjorken variable and $Q^{2}$ scales and cannot be used to construct the data points even utilizing the linear interpolation. Nonetheless, there are limited data with the identical bins of $x$ and $Q^{2}$ and few others which are binned not so much differently with respect to the $x$ and $Q^{2}$ such that the linear interpolation method is applicable for them. These data are selected such as to be placed in the inelastic region. In fact, these data are selected such that they exclude the elastic and $\Delta$ resonance particles. They are depicted in three different panels in Fig. $4{ }^{1}$

\section{V. $Q^{2}$ DEPENDENCE OF BJORKEN SUM RULE IN ANQCD APPROACH}

Here we investigate the Bjorken sum rule (BSR) [52] in the $2 \delta$ anQCD model. This sum rule is relating the spin dependence of quark densities to the axial charge. The BSR is important to understand the nucleon spin structure, that QCD can describe well the strong force in the polarized case; i.e., its $Q^{2}$ dependence reflects the strong force in the polarized case. BSR has been measured at SLAC, DESY, and CERN [45,46,50,51,53-63] via a polarized deep inelastic lepton scattering process. It has also been measured at moderate values of $Q^{2}$ by Jefferson Lab (JLab) [64-67]. The $Q^{2}$ momentum that is probing the nucleon is related to the inverse of the space-time scale. BSR at high $Q^{2}$ was evaluated in conventional pQCD by Refs. [68-71] and has the form

$$
\begin{aligned}
\Gamma_{1}^{p-n} \equiv & \int_{0}^{1} d x\left(g_{1}^{p}\left(x, Q^{2}\right)-g_{1}^{n}\left(x, Q^{2}\right)\right) \\
= & \frac{g_{A}}{6}\left[1-\frac{\alpha_{s}}{\pi}-d_{1} \frac{\alpha_{s}^{2}}{\pi^{2}}-d_{2} \frac{\alpha_{s}^{3}}{\pi^{3}}+d_{3} \frac{\alpha_{s}^{4}}{\pi^{4}}+\cdots\right] \\
& +\sum_{i=2}^{\infty} \frac{\mu_{2 i}^{p-n}\left(Q^{2}\right)}{Q^{2 i-2}} .
\end{aligned}
$$

In this equation, the spin-dependent proton and neutron structure functions are $g_{1}^{p}$ and $g_{1}^{n}$, respectively. The strength of the neutron $\beta$ decay is controlling by $g_{A}$, which is the nucleon axial charge. Soft gluon radiation in conventional pQCD causes the leading-twist term (known as $\mu_{2}$ ), given by the first term in the right-hand side of Eq. (50), to

\footnotetext{
${ }^{1}$ We thank S. E. Kühn for useful comments on this point.
}

depend smoothly on $Q^{2}$. The $d_{i}$ coefficients up to $\alpha_{s}^{4}$ order can be found in Ref. [68]. The terms with $\mu_{4}, \mu_{6}$, etc., are the nonperturbative power corrections, usually known as higher twists (HT). The higher twists, in fact, reflect a correlation between quarks and gluons. For a good understanding of the nucleon structure at the low-energy scales, analyzing the HT is essential. This is why it is interesting to follow as well this subject in the anQCD approaches, as we mentioned in the introduction. We recall that, in the introductory part, a brief review has been done independent of the specific scheme. Therefore, the references cited here are not also in the Lambert scheme (usually, they are in the $\overline{\mathrm{MS}}$ scheme), but the evaluations here are in the $\left(c_{2}=-4.9\right)$ Lambert scheme.

The anQCD modification of the BSR, using the $2 \delta$ anQCD model, has the form

$$
\Gamma_{1}^{p-n}=\Gamma_{1, \text { anQCD }}^{p-n}+\sum_{i=2}^{\infty} \frac{\mu_{2 i}^{\mathrm{anQCD}}\left(Q^{2}\right)}{Q^{2 i-2}},
$$

where

$$
\Gamma_{1, \mathrm{anQCD}}^{p-n}=\frac{g_{A}}{6}\left[1-\Delta_{1, \mathrm{anQCD}}^{p-n}\left(Q^{2}\right)\right]
$$

and

$$
\Delta_{1, \text { anQCD }}^{p-n}=d_{1}(k) \mathcal{A}_{1}+d_{2}(k) \mathcal{A}_{2}+d_{3}(k) \mathcal{A}_{3}+d_{4}(k) \mathcal{A}_{4},
$$

where $d_{i}(k)$ coefficients are in the Lambert scheme with $c_{2}=-4.9$ (cf. Appendix A in Ref. [72]). The $k$ parameter is the renormalization scale parameter $k=\mu^{2} / Q^{2}$, the couplings are $\mathcal{A}_{n}=\mathcal{A}_{n}^{(2 \delta)}\left(\mu^{2}\right)$, and we fixed the value of $k$ to $k=1$.

The higher-twist effects are included in Eq. (51). The term with dimension $D=2$, i.e., $\mu_{4}^{p-n} / Q^{2}$, has the following coefficient [73]:

$$
\mu_{4}=\frac{M_{N}^{2}}{9}\left(a_{2}^{p-n}+4 d_{2}^{p-n}+4 f_{2}^{p-n}\left(Q^{2}\right)\right)
$$

In this equation, the nucleon mass is $M_{N} \approx 0.94 \mathrm{GeV}$. The coefficient $a_{2}^{p-n}$ represents the twist-2 target mass correction; $d_{2}^{p-n}$ is related to the twist-3 matrix element [73]. These coefficients can be computed, using the following relations [73]:

$$
\begin{aligned}
& a_{2}^{p-n}=\int_{0}^{1} d x x^{2} g_{1}^{p-n} \\
& d_{2}^{p-n}=\int_{0}^{1} d x x^{2}\left(2 g_{1}^{p-n}+3 g_{2}^{p-n}\right) .
\end{aligned}
$$

To calculate $d_{2}^{p-n}$, we need the $g_{2}$ structure function. However, there is a relation which gives us $g_{2}$ in terms of the $g_{1}$ structure function in Ref. [74], but this relation 
includes just the twist-2 part of $g_{2}$ and it cannot be substituted in Eq. (53), since it is related to the twist-3 matrix element. Instead, one can resort to the quoted value for $d_{2}^{p-n}$ in Refs. [48,67], a result from recent experimental analysis. The numerical value which is reported there is $d_{2}^{p-n}=0.008 \pm 0.0036$.

Since one can compute the polarized structure functions $g_{1}^{p}$ and $g_{1}^{n}$ [see Eq. (43)], it is possible to obtain the numerical values for $a_{2}^{p-n}$ in Eq. (53) at $Q^{2}=1 \mathrm{GeV}^{2}$, i.e., $a_{2}^{p-n}=0.0157 \pm 0.0001$. To calculate the polarized structure function $g_{1}^{\mathrm{NS}}$, it is required at first to achieve the polarized valence densities at initial scale $Q_{0}$, given by Eq. (31). The unknown parameters of these densities can be obtained using the traditional global fit which has been done in Refs. [37,40]. It should be recalled that the polarized structure function $g_{1}^{\mathrm{NS}}$ can be calculated, based on the formalism which we applied in this article. But the numerical values of the unknown parameters for the required parton densities have been quoted from Refs. [37,40], listed in Table I.

The $f_{2}^{p-n}$ function in Eq. (52) is the expectation value of a well-defined operator with a specific physical meaning $[75,76]$. Here it is regarded as a parameter which parameterizes a power correction to the anQCD analysis. From this point of view, we take the anQCD evolution form (based on its pQCD form) such that $f_{2}^{p-n}(Q)=$ $f_{2}^{p-n}\left(Q_{0}\right) \mathcal{A}_{\gamma_{0} / 8 \beta_{0}}^{(2 \delta)}\left(Q^{2}\right) / \mathcal{A}_{\gamma_{0} / 8 \beta_{0}}^{(2 \delta)}\left(Q_{0}^{2}\right)$ as has been quoted in Ref. [72].

While $f_{2}^{p-n}$ is evaluated in the $2 \delta$ anQCD approach, the coefficients $a_{2}$ and $d_{2}$, as twist- 2 and twist- 3 quantities, are obtained from the underlying $\mathrm{pQCD}$ and the analysis of related experimental data, respectively. In fact, the first integral in Eq. (53) can be calculated from the computed polarized structure function $g_{1}^{\mathrm{NS}}$. As we explained in the two previous paragraphs, to compute it one needs to use the traditional global fit as in Refs. [37,40] to obtain the unknown parameters of the polarized valence densities in Eq. (31).

We extend our computations up to the fourth higher twist to include as well the $\mu_{6}$ term. In our calculations, we have in total two free parameters $\mu_{6}$ and $f_{2}^{p-n}\left(Q_{0}\right)$, which can be obtained by fitting to the available experimental data for the BSR. The fitted numerical values are $\mu_{6}=0.0007 \pm$ 0.0001 and $f_{2}^{p-n}\left(Q_{0}\right)=-0.020 \pm 0.001$, respectively. Based on Eq. (52), the numerical value for $\mu_{4}$ would be $\mu_{4}=-0.0031 \pm 0.001$.

Equation (51) is the analytical result for $\Gamma_{1}^{p-n}$ in the $2 \delta$ anQCD model. In Fig. 5, we present $\Gamma_{1}^{p-n}$ in the $2 \delta \mathrm{anQCD}$ approach and compare with the result from the underlying $\mathrm{pQCD}$ where both are considered in the Lambert scheme, as well as with the E143, E154, and E155 [50,53,63] and JLab [64-67] experimental data. The good agreement between the available experimental data and the analytical result from the $2 \delta$ anQCD model, in contrast to

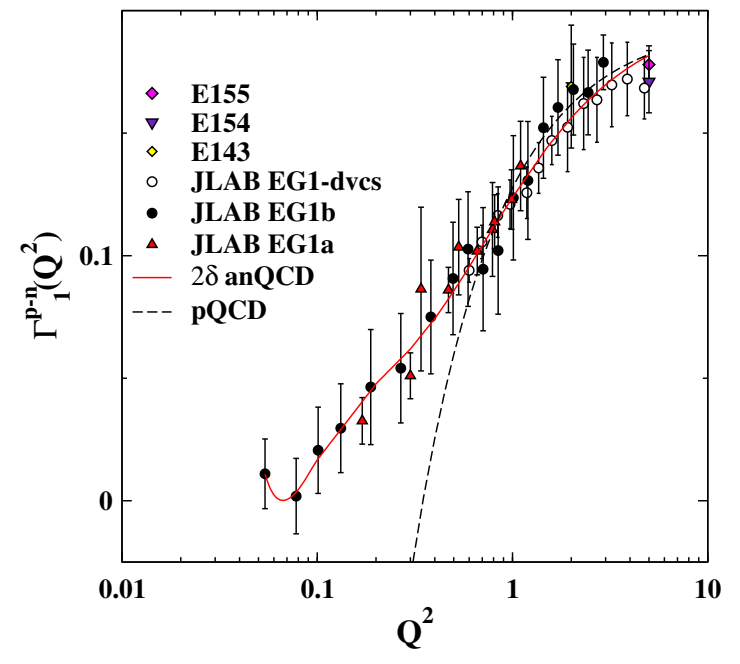

FIG. 5. The BSR, $\Gamma_{1}^{p-n}\left(Q^{2}\right)$, resulted from two models. The red line gives the result from $2 \delta$ anQCD model, and the black line from the underlying $\mathrm{pQCD}$. Other symbols show the data from the E143, E154, and E155 [50,53,63] and JLab [64-67] experiments.

the underlying pQCD result especially at low energies, confirms that the anQCD approach, based on the employed model, is working well.

\section{SUMMARY AND CONCLUSION}

As a new theoretical approach to analyze the nonsinglet polarized structure function at the low- $Q^{2}$ continuum region, the analytic QCD (anQCD), specifically the $2 \delta$ anQCD model, is employed to evaluate this function and also the $Q^{2}$ dependence of the Bjorken sum rule.

Using this approach, it is possible to analyze the polarized structure function in the whole $Q^{2}$ range at the leading-twist order. On this basis, one may conclude some outstanding characteristics arising from the anQCD approach. At first, it can be shown that the $g_{1}^{(\mathrm{NS})}\left(x, Q^{2}\right)$ structure function at a fixed $x$ value and in the whole range of $Q^{2}$ is slowly changing. The plot in Fig. 2 reveals this feature. Second, the results of the underlying $\mathrm{pQCD}$ and anQCD approaches at moderate and high $Q^{2}$ scales are consistent with each other as can be seen from the depicted plots in Fig. 3. Third, at low values of $Q^{2}$, the evolution of $g_{1}^{(\mathrm{NS})}\left(x, Q^{2}\right)$ as a function of $x$ in the anQCD approach is slower and smoother in comparison with the underlying pQCD which are both in the Lambert scheme. This fact can be seen from the three plots in Fig. 4 at the low-energy scales $Q^{2}=0.2,0.17$, and $0.07 \mathrm{GeV}^{2}$, respectively. Consequently, we conclude that the result from analytic series in the anQCD approach, using the $2 \delta$ anQCD model, better reproduces the data and behaves more smoothly than in underlying pQCD, especially at low $Q^{2}$ values.

We also investigated the $Q^{2}$ dependence of the Bjorken sum rule in the anQCD approach, using the $2 \delta$ anQCD 
model. The result is shown in Fig. 5, which indicates good agreement with the available experimental data in the whole interval of energy scales. The agreement between them especially at the low-energy scales confirms the validity of the employed anQCD approach. A comparison has also been done with the result from the underlying pQCD approach, suggesting an advantage of the anQCD over the pQCD approach in the evaluation of spacelike QCD observables at low energies.

All these features have their origin in the fact that the behavior of analytic (holomorphic) coupling is under control in the entire range of spacelike $Q^{2}$ values, including at $Q^{2} \mapsto 0$. This outstanding feature has been presented in Fig. 1.

As a final point, we mention that the plots in Fig. 2, toward small values of $Q^{2}$, resulted from Eq. (46) in the $2 \delta$ anQCD model. In this case, the Jacobi polynomial expansion is used [see Eq. (43)] to convert the result from the moment space to $x$-Bjorken space. In Eq. (43), the notation $\Delta M\left[x g_{1}^{\mathrm{NS}}, j+2\right]$ for the polarized moment of structure function is equivalent to the $\Delta \mathcal{M}_{\mathrm{NS}}\left(n, Q^{2}\right)$ in Eq. (46), with $n=j+2$. It should be noted that, in converting the structure function from the moment space to Bjorken- $x$ space in underlying pQCD, we used the inverse Mellin technique based on Eq. (44), while for the anQCD approach, due to computational difficulties which arise in employing the inverse Mellin technique, we resorted to employing the technique of Jacobi polynomial expansion.

A further research task and an interesting subject would be to extend the anQCD analysis to the nonsinglet case for the unpolarized structure function which contains heavy quark flavors. In this case, the effect of quark mass cannot be ignored, and it should be considered in the calculations. In this regard, the related commands in the $2 \delta$ anQCD model would be different with respect to what we used here for light quarks. Also of interest would be to consider the singlet case of structure functions in the anQCD approach, a subject on which we hope to report in the future.

\section{ACKNOWLEDGMENTS}

The authors are indebted to G. Cvetič and C. Ayala for reading the manuscript and providing crucial comments which helped us improve the manuscript. We are grateful to S. E. Kühn for the very essential discussions about the CLAS data. We also acknowledge G. Mallot for productive comments and providing us with the required COMPASS data. We are finally thankful to Charles C. Young for his useful comments in connection to the related CERN data. S.A.T. is grateful to the School of Particles and Accelerators, Institute for Research in Fundamental Sciences (IPM) to make the required facilities to do this project.

\section{APPENDIX: THE SPLITTING FUNCTIONS IN THE NLO APPROXIMATION}

The analytical expression for the splitting functions in the moment space for nonsinglet sectors and at the NLO approximation are taken from Ref. [34] and presented below. The usual quadratic Casimir operators are fixed to their exact values, using $C_{A}=3, T_{F}=\frac{1}{2}$, and $C_{F}=\frac{4}{3}$ :

$$
\begin{aligned}
-\Delta P_{\mathrm{NS} \pm}^{(1) n}= & C_{F}^{2}\left[2 \frac{2 n+1}{n^{2}(n+1)^{2}} S_{1}(n)+2\left(2 S_{1}(n)-\frac{1}{n(n+1)}\right)\left(S_{2}(n)-S_{2}^{\prime}\left(\frac{n}{2}\right)\right)\right. \\
& \left.+3 S_{2}(n)+8 \tilde{S}(n)-S_{3}^{\prime}\left(\frac{n}{2}\right)-\frac{3 n^{3}+n^{2}-1}{n^{3}(n+1)^{3}}-\frac{3}{8} \mp 2 \frac{2 n^{2}+2 n+1}{n^{3}(n+1)^{3}}\right] \\
& +C_{F} C_{A}\left[\frac{67}{9} S_{1}(n)-\left(2 S_{1}(n)-\frac{1}{n(n+1)}\right)\left(2 S_{2}(n)-S_{2}^{\prime}\left(\frac{n}{2}\right)\right)-\frac{11}{3} S_{2}(n)-4 \tilde{S}(n)+\frac{1}{2} S_{3}^{\prime}\left(\frac{n}{2}\right)\right. \\
& \left.-\frac{1}{18} \frac{151 n^{4}+236 n^{3}+88 n^{2}+3 n+18}{n^{3}(n+1)^{3}}-\frac{17}{24} \pm \frac{2 n^{2}+2 n+1}{n^{3}(n+1)^{3}}\right] \\
& +C_{F} T_{f}\left[-\frac{20}{9} S_{1}(n)+\frac{4}{3} S_{2}(n)+\frac{2}{9} \frac{11 n^{2}+5 n-3}{n^{2}(n+1)^{2}}+\frac{1}{6}\right], .
\end{aligned}
$$

where

$$
\begin{gathered}
S_{k}(n) \equiv \sum_{j=1}^{n} \frac{1}{j^{k}}, \\
S_{k}^{\prime}\left(\frac{n}{2}\right) \equiv 2^{k-1} \sum_{j=1}^{n} \frac{1+(-)^{j}}{j^{k}}=\frac{1}{2}(1+\eta) S_{k}\left(\frac{n}{2}\right)+\frac{1}{2}(1-\eta) S_{k}\left(\frac{n-1}{2}\right),
\end{gathered}
$$




$$
\begin{gathered}
\tilde{S}(n) \equiv \sum_{j=1}^{n} \frac{(-)^{j}}{j^{2}} S_{1}(j)=-\frac{5}{8} \zeta(3)+\eta\left[\frac{S_{1}(n)}{n^{2}}+\frac{\pi^{2}}{12} G(n)+\int_{0}^{1} d x x^{n-1} \frac{\mathrm{Li}_{2}(x)}{1+x}\right] \\
\frac{\mathrm{Li}_{2}(x)}{(1+x)} \equiv\left(1.01 x-0.846 x^{2}+1.155 x^{3}-1.074 x^{4}+0.55 x^{5}\right) .
\end{gathered}
$$

In Eq. (A4), $G(n)$ is defined as $G(n) \equiv \psi\left(\frac{n+1}{2}\right)-\psi\left(\frac{n}{2}\right)$, where $\psi(z)=d \ln \Gamma(z) / d z$. For $\Delta P_{\mathrm{NS} \pm}^{(1) n}$, it is assumed $\eta= \pm 1$, while for anomalous dimensions of nonsinglet flavor, $\eta=+1$ is considered.

The $S_{k}(n)$ functions can be written in terms of the harmonic sums such as $[77,78]$

$$
\begin{aligned}
& S_{1}(n)=\gamma_{E}+\psi(n+1), \\
& S_{2}(n)=\zeta(2)-\psi^{\prime}(n+1), \\
& S_{3}(n)=\zeta(3)+0.5 \psi^{\prime \prime}(n+1),
\end{aligned}
$$

where $\gamma_{E}=0.577216$ is the Euler constant, $\zeta(2)=\pi^{2} / 6$, and $\zeta(3)=1.20206$.

[1] D. V. Shirkov and I. L. Solovtsov, Analytic Model for the QCD Running Coupling with Universal $\alpha_{s}(0)$ Value, Phys. Rev. Lett. 79, 1209 (1997).

[2] K. A. Milton and I. L. Solovtsov, Analytic perturbation theory in QCD and Schwinger's connection between the beta function and the spectral density, Phys. Rev. D 55, 5295 (1997).

[3] K. A. Milton, I. L. Solovtsov, and O. P. Solovtsova, Analytic perturbation theory and inclusive tau decay, Phys. Lett. B 415, 104 (1997).

[4] K. A. Milton and O.P. Solovtsova, Analytic perturbation theory: A new approach to the analytic continuation of the strong coupling constant $\alpha_{s}$ into the timelike region, Phys. Rev. D 57, 5402 (1998).

[5] D. V. Shirkov, Renormalization group, causality and nonpower perturbation expansion in QFT, Theor. Math. Phys. 119, 438 (1999).

[6] D. V. Shirkov, Analytic perturbation theory for QCD observables, Theor. Math. Phys. 127, 409 (2001).

[7] C. Ayala, C. Contreras, and G. Cvetič, Extended analytic QCD model with perturbative QCD behavior at high momenta, Phys. Rev. D 85, 114043 (2012).

[8] C. Ayala and G. Cvetič, anQCD: A Mathematica package for calculations in general analytic QCD models, Comput. Phys. Commun. 190, 182 (2015).

[9] S. J. Brodsky, G. F. de Teramond, and A. Deur, Nonperturbative QCD Coupling and its $\beta$-function from light-front holography, Phys. Rev. D 81, 096010 (2010).

[10] Y. L. Dokshitzer, G. Marchesini, and B. R. Webber, Dispersive approach to power behaved contributions in QCD hard processes, Nucl. Phys. B469, 93 (1996).

[11] Y. L. Dokshitzer and B. R. Webber, Calculation of power corrections to hadronic event shapes, Phys. Lett. B 352, 451 (1995).
[12] A. Deur, S. J. Brodsky, and G. F. de Teramond, The QCD running coupling, Prog. Part. Nucl. Phys. 90, 1 (2016).

[13] A. I. Karanikas and N. G. Stefanis, Analyticity and power corrections in hard scattering hadronic functions, Phys. Lett. B 504, 225 (2001); Erratum, Phys. Lett. B 636, 330(E) (2006).

[14] A. P. Bakulev, S. V. Mikhailov, and N. G. Stefanis, QCD analytic perturbation theory: From integer powers to any power of the running coupling, Phys. Rev. D 72, 074014 (2005); Erratum, Phys. Rev. D 72, 119908(E) (2005).

[15] A. P. Bakulev, S. V. Mikhailov, and N. G. Stefanis, Fractional analytic perturbation theory in Minkowski space and application to Higgs boson decay into a $b \bar{b}$ pair, Phys. Rev. D 75, 056005 (2007); Erratum, Phys. Rev. D 77, 079901(E) (2008).

[16] A. P. Bakulev, S. V. Mikhailov, and N. G. Stefanis, Higherorder QCD perturbation theory in different schemes: From FOPT to CIPT to FAPT, J. High Energy Phys. 06 (2010) 085 .

[17] A. P. Bakulev, Global fractional analytic perturbation theory in QCD with selected applications, Phys. Part. Nucl. 40, 715 (2009).

[18] C. Ayala and S. V. Mikhailov, How to perform QCD analysis of DIS in analytic perturbation theory, Phys. Rev. D 92, 014028 (2015); N. G. Stefanis, Taming Landau singularities in QCD perturbation theory: The analytic approach, Phys. Part. Nucl. 44, 494 (2013).

[19] A. V. Sidorov and O. P. Solovtsova, The QCD analysis of the combined set for the $F_{3}$ structure function data based on the analytic approach, Mod. Phys. Lett. A 29, 1450194 (2014).

[20] A. V. Nesterenko, Quark-anti-quark potential in the analytic approach to QCD, Phys. Rev. D 62, 094028 (2000); New analytic running coupling in space-like and time-like regions, Phys. Rev. D 64, 116009 (2001). 
[21] A. V. Nesterenko and J. Papavassiliou, The massive analytic invariant charge in QCD, Phys. Rev. D 71, 016009 (2005); A. C. Aguilar, A. V. Nesterenko, and J. Papavassiliou, Infrared enhanced analytic coupling and chiral symmetry breaking in QCD, J. Phys. G 31, 997 (2005); A. V. Nesterenko and J. Papavassiliou, A novel integral representation for the Adler function, J. Phys. G 32, 1025 (2006); A. V. Nesterenko, eConf C0706044, 25 (2007).

[22] A. I. Alekseev, Synthetic running coupling of QCD, Few-Body Syst. 40, 57 (2006).

[23] Y. Srivastava, S. Pacetti, G. Pancheri, and A. Widom, Dispersive techniques for $\alpha_{s}, R_{\text {had }}$ and instability of the perturbative vacuum, eConf C010430, T19 (2001).

[24] B. R. Webber, QCD power corrections from a simple model for the running coupling, J. High Energy Phys. 10 (1998) 012.

[25] G. Cvetič and C. Valenzuela, An approach for evaluation of observables in analytic versions of QCD, J. Phys. G 32, L27 (2006).

[26] G. Cvetič and C. Valenzuela, Various versions of analytic QCD and skeleton-motivated evaluation of observables, Phys. Rev. D 74, 114030 (2006); Erratum, Phys. Rev. D 84, 019902(E) (2011).

[27] G. M. Prosperi, M. Raciti, and C. Simolo, On the running coupling constant in QCD, Prog. Part. Nucl. Phys. 58, 387 (2007).

[28] G. Cvetič and C. Valenzuela, Analytic QCD: A short review, Braz. J. Phys. 38, 371 (2008).

[29] A. P. Bakulev and V. L. Khandramai, FAPT: A Mathematica package for calculations in QCD fractional analytic perturbation theory, Comput. Phys. Commun. 184, 183 (2013).

[30] G. Cvetič and A. V. Kotikov, Analogs of noninteger powers in general analytic QCD, J. Phys. G 39, 065005 (2012).

[31] G. Cvetič, Techniques of evaluation of QCD low-energy physical quantities with running coupling with infrared fixed point, Phys. Rev. D 89, 036003 (2014).

[32] A. V. Kotikov, V. G. Krivokhizhin, and B. G. Shaikhatdenov, Analytic and 'frozen' QCD coupling constants up to NNLO from DIS data, Phys. At. Nucl. 75, 507 (2012).

[33] C. Ayala and G. Cvetič, Mathematica and Fortran programs for various analytic QCD couplings, J. Phys. Conf. Ser. 608, 012064 (2015).

[34] B. Lampe and E. Reya, Spin physics and polarized structure functions, Phys. Rep. 332, 1 (2000).

[35] T. Van Ritbergen, J. A. M. Vermaseren, and S. A. Larin, The four-loop beta-function in quantum chromodynamics, Phys. Lett. B 400, 379 (1997).

[36] F. J. Yndurain, The Theory of Quarks and Gluons Interactions, 4th ed. (Springer-Verlag, Berlin, 2006).

[37] A. N. Khorramian, S. Atashbar Tehrani, S. Taheri Monfared, F. Arbabifar, and F. I. Olness, Polarized deeply inelastic scattering (DIS) structure functions for nucleons and nuclei, Phys. Rev. D 83, 054017 (2011).

[38] M. Tanabashi et al. (Particle Data Group), Review of particle physics, Phys. Rev. D 98, 030001 (2018).

[39] A. L. Kataev and A. V. Sidorov, The Jacobi polynomials QCD analysis of the CCFR data for $x F_{3}$ and the $Q^{2}$ dependence of the Gross-Llewellyn-Smith sum rule, Phys. Lett. B 331, 179 (1994).
[40] H. Khanpour, S. T. Monfared, and S. A. Tehrani, Study of spin-dependent structure functions of ${ }^{3} \mathrm{He}$ and ${ }^{3} \mathrm{H}$ at NNLO approximation and corresponding nuclear corrections, Phys. Rev. D 96, 074037 (2017).

[41] H. Khanpour, S. T. Monfared, and S. A. Tehrani, Nucleon spin structure functions at NNLO in the presence of target mass corrections and higher twist effects, Phys. Rev. D 95, 074006 (2017).

[42] F. Taghavi-Shahri, Z. A. Yazdi, and S. A. Tehrani, Next-tonext-to-leading order calculation of valence quark polarization in the valon model, Int. J. Theor. Phys. 58, 157 (2019).

[43] G. Parisi and N. Sourlas, A simple parametrization of the $Q^{2}$ dependence of the quark distributions in QCD, Nucl. Phys. B151, 421 (1979); I. S. Barker, C. S. Langensiepen, and G. Shaw, General parametrization of scale breaking, Nucl. Phys. B186, 61 (1981).

[44] A. Vogt, Efficient evolution of unpolarized and polarized parton distributions with QCD-PEGASUS, Comput. Phys. Commun. 170, 65 (2005); M. Glück, E. Reya, and A. Vogt, Radiatively generated parton distributions for high-energy collisions, Z. Phys. C 48, 471 (1990).

[45] A. Airapetian, N. Akopov, Z. Akopov, A. Andrus, E. C. Aschenauer, W. Augustyniak, R. Avakian, A. Avetissian, E. Avetissian, S. Belostotski et al. (HERMES Collaboration), Precise determination of the spin structure function $g_{1}$ of the proton, deuteron and neutron, Phys. Rev. D 75, 012007 (2007).

[46] B. Adeva, T. Akdogan, E. Arik, A. Arvidson, B. Badelek, G. Bardin, G. Baum, P. Berglund, L. Betev, I. G. Bird et al. (Spin Muon Collaboration), Spin asymmetries $A_{1}$ and structure functions $g_{1}$ of the proton and the deuteron from polarized high-energy muon scattering, Phys. Rev. D 58, 112001 (1998).

[47] C. Adolph et al. (COMPASS Collaboration), The spin structure function $g_{1}^{\mathrm{p}}$ of the proton and a test of the Bjorken sum rule, Phys. Lett. B 753, 18 (2016).

[48] A. Deur, S. J. Brodsky, and G. F. Teramond, The spin structure of the nucleon, Rep. Prog. Phys. 82, 076201 (2019).

[49] S.E. Kühn et al. (CLAS Collaboration) (private communication).

[50] K. Abe, T. Akagi, P. L. Anthony, R. Antonov, R. G. Arnold, T. Averett, H. R. Band, J. M. Bauer, H. Borel, P. E. Bosted et al. (E143 Collaboration), Measurements of the proton and deuteron spin structure functions $g_{1}$ and $g_{2}$, Phys. Rev. D 58, 112003 (1998).

[51] K. Ackerstaff, A. Airapetian, I. Akushevich, N. Akopov, M. Amarian, E. C. Aschenauer, R. Avakian, H. Avakian, A. Avetissian, B. Bains et al. (HERMES Collaboration), Measurement of the neutron spin structure function $g_{1}^{n}$ with a polarized ${ }^{3} \mathrm{He}$ internal target, Phys. Lett. B 404, 383 (1997).

[52] J. D. Bjorken, Applications of the chiral U(6) x (6) algebra of current densities, Phys. Rev. 148, 1467 (1966); Inelastic scattering of polarized leptons from polarized nucleons, Phys. Rev. D 1, 1376 (1970).

[53] K. Abe, T. Akagi, B. D. Anderson, P. L. Anthony, R. G. Arnold, T. Averett, H. R. Band, C. M. Berisso, P. Bogorad, H. Borel et al. (E154 Collaboration), Next-to-leading order 
QCD analysis of polarized deep inelastic scattering data, Phys. Lett. B 405, 180 (1997).

[54] K. Abe, T. Akagi, B. D. Anderson, P. L. Anthony, R. G. Arnold, T. Averett, H. R. Band, C. M. Berisso, P. Bogorad, H. Borel et al. (E154 Collaboration), Precision Determination of the Neutron Spin Structure Function $g_{1}(n)$, Phys. Rev. Lett. 79, 26 (1997).

[55] P. L. Anthony, R. G. Arnold, T. Averette, H. R. Band, M. C. Berisso, H. Borel, P.E. Bosted, S. L. Bültmann, M. Buenerd, T. E. Chupp et al. (E155 Collaboration), Measurement of the proton and deuteron spin structure functions $g_{2}$ and asymmetry $A_{2}$, Phys. Lett. B 458, 529 (1999).

[56] P. L. Anthony, R. G. Arnold, T. Averette, H. R. Band, M. C. Berisso, H. Borel, P. E. Bosted, S. L. Bültmann, M. Buenerd, T. E. Chupp et al. (E155 Collaboration), Measurement of the deuteron spin structure function $g_{1}^{d}(x)$ for $1-(\mathrm{GeV} / c)^{2}<Q^{2}<40-(\mathrm{GeV} / c)^{2}$, Phys. Lett. B 463, 339 (1999).

[57] P. L. Anthony, R. G. Arnold, T. Averette, H. R. Band, M. C. Berisso, H. Borel, P. E. Bosted, S. L. Bültmann, M. Buenerd, T. E. Chupp et al. (E155 Collaboration), Measurements of the $Q^{2}$ dependence of the proton and neutron spin structure functions $g_{1}^{p}$ and $g_{1}^{n}$, Phys. Lett. B 493, 19 (2000).

[58] P. L. Anthony, R. G. Arnolda, T. Averett, H. R. Band, N. Benmouna, W. Boeglin, H. Borel, P. E. Bosted, S. L. Bültmann, G. R. Court et al. (E155 Collaboration), Precision measurement of the proton and deuteron spin structure functions $g_{2}$ and asymmetries $A_{2}$, Phys. Lett. B 553, 18 (2003).

[59] K. Ackerstaff, A. Airapetian, I. Akushevich, N. Akopov, M. Amarian, E. C. Aschenauer, R. Avakian, H. Avakian, A. Avetissian, B. Bains et al. (HERMES Collaboration), Determination of the deep inelastic contribution to the generalized Gerasimov-Drell-Hearn integral for the proton and neutron, Phys. Lett. B 444, 531 (1998).

[60] K. Ackerstaff, A. Airapetian, I. Akushevich, N. Akopov, M. Amarian, E. C. Aschenauer, R. Avakian, H. Avakian, A. Avetissian, B. Bains et al. (HERMES Collaboration), Measurement of the proton spin structure function $g_{1}^{p}$ with a pure hydrogen target, Phys. Lett. B 442, 484 (1998).

[61] A. Airapetian, N. Akopov, Z. Akopov, M. Amarian, V. V. Ammosov, E. C. Aschenauer, H. Avakian, R. Avakian, A. Avetissian, E. Avetissian et al. (HERMES Collaboration), Evidence for Quark Hadron Duality in the Proton Spin Asymmetry $A_{1}$, Phys. Rev. Lett. 90, 092002 (2003).

[62] A. Airapetian, N. Akopov, Z. Akopov, M. Amarian, V. V. Ammosov, E. C. Aschenauer, R. Avakian, A. Avetissian, E. Avetissian, P. Bailey et al. (HERMES Collaboration), The $Q^{2}$ dependence of the generalized Gerasimov-Drell-Hearn integral for the deuteron, proton and neutron, Eur. Phys. J. C 26, 527 (2003).
[63] P. E. Bosted (E155 and E155x Collaborations), Spin physics experiments at SLAC, Int. J. Mod. Phys. A 18, 1169 (2003).

[64] A. Deur, P. Bosted, V. Burkert, G. Cates, J.-P. Chen, S. Choi, D. Crabb, C. W. de Jager, R. De Vita, G. E. Dodge et al., Experimental Determination of the Evolution of the Bjorken Integral at Low $Q^{2}$, Phys. Rev. Lett. 93, 212001 (2004).

[65] A. Deur, P. Bosted, V. Burkert, D. Crabb, V. Dharmawardane, G. E. Dodge, T. A. Forest, K. A. Griffioen, S. E. Kuhn, R. Minehart, and Y. Prok, Experimental study of isovector spin sum rules, Phys. Rev. D 78, 032001 (2008).

[66] J. P. Chen, A. Deur, and Z. E. Meziani, Sum rules and moments of the nucleon spin structure functions, Mod. Phys. Lett. A 20, 2745 (2005).

[67] A. Deur, Y. Prok, V. Burkert, D. Crabb, F.-X. Girod, K. A. Griffioen, N. Guler, S. E. Kuhn, and N. Kvaltine, High precision determination of the $Q^{2}$ evolution of the Bjorken sum, Phys. Rev. D 90, 012009 (2014).

[68] P. A. Baikov, K. G. Chetyrkin, and J. H. Kühn, Adler Function, Bjorken Sum Rule, and the Crewther Relation to Order $\alpha_{s}^{4}$ in a General Gauge Theory, Phys. Rev. Lett. 104, 132004 (2010).

[69] A. L. Kataev, The Ellis-Jaffe sum rule: The Estimates of the next to next-to-leading order QCD corrections, Phys. Rev. D 50, R5469 (1994).

[70] A. L. Kataev, Deep inelastic sum rules at the boundaries between perturbative and nonperturbative QCD, Mod. Phys. Lett. A 20, 2007 (2005).

[71] R. S. Pasechnik, D. V. Shirkov, O. V. Teryaev, O. P. Solovtsova, and V. L. Khandramai, Nucleon spin structure and pQCD frontier on the move, Phys. Rev. D 81, 016010 (2010).

[72] C. Ayala, G. Cvetič, A. V. Kotikov, and B. G. Shaikhatdenov, Bjorken polarized sum rule and infrared-safe QCD couplings, Eur. Phys. J. C 78, 1002 (2018).

[73] E. V. Shuryak and A. I. Vainshtein, Theory of power corrections to deep inelastic scattering in quantum chromodynamics 2. $Q^{4}$ effects: Polarized target, Nucl. Phys. B201, 141 (1982).

[74] S. Wandzura and F. Wilczek, Sum rules for spin dependent electroproduction: Test of relativistic constituent quarks, Phys. Lett. 72B, 195 (1977).

[75] M. Burkardt, Transverse force on quarks in deep-inelastic scattering, Phys. Rev. D 88, 114502 (2013).

[76] M. Abdallah and M. Burkardt, Transverse force on transversely polarized quarks in longitudinally polarized nucleons, Phys. Rev. D 94, 094040 (2016).

[77] J. A. M. Vermaseren, Harmonic sums, Mellin transforms and integrals, Int. J. Mod. Phys. A 14, 2037 (1999).

[78] J. Blümlein and S. Kurth, Harmonic sums and Mellin transforms up to two loop order, Phys. Rev. D 60, 014018 (1999). 